\title{
Stability of Multilayer Extrusion of Viscoelastic Liquids
}

A linear stability analysis of multilayer plane Poiseuille flow of Oldroyd-B liquids with shear rate dependent viscosities is performed for an arbitrary number of layers. Asymptotic solutions at long wavelengths and numerical solutions at wavelengths of $O(1)$ are obtained for two-dimensional infinitesimal disturbances. The asymptotic solutions are identical for viscoelastic and Newtonian liquids in two- and threelayer flows, except for nearly geometrically symmetric configurations in N. R. Anturkar
T. C. Papanastasiou
J. O. Wilkes
Department of Chemical Engineering
University of Michigan
Ann Arbor, Ml 48109 three-layer flows. Multilayer flows of viscoelastic liquids can be stable at all wavelengths; thus, operating diagrams of stable flows can be constructed. Symmetric and nearly symmetric configurations in threelayer flows are unstable when the core layer is more viscous than the outer layers. For highly elastic liquids, stability is not influenced by elasticity, whereas shear thinning always destabilizes the flow. The analysis provides guidelines to avoid interfacial instabilities, which originate inside dies of multilayer extrusion.

\section{Introduction}

In the polymer industry, multilayer sheets and films are produced by coextrusion and utilized as products of improved optical and physical properties. Each layer of these composite materials provides a specific end-use characteristic, such as oxygen and moisture barrier, thermoformability, and mechanical properties. Applications of such multilayer films range from food packaging to laminated papers. At certain operating conditions, wavy interfaces are observed in the multilayer flow, a phenomenon known as interfacial instability. In extreme cases, this instability can cause intermixing of layers and is detrimental to the quality of the product (Han, 1981). These instabilities were shown to originate inside the die, where multilayer Poiseuille-like flow prevails (Schrenk and Alfrey, 1978).

The phenomenon of interfacial instability in two-layer plane Poiseuille flow of Newtonian liquids was investigated by Yih (1967) using linear stability analysis. He found that the eigenvalues in multilayer flows consist of an interfacial mode arising from the presence of the interface, and that at low speeds the interfacial mode is the only one that may become unstable. Yih considered long-wavelength asymptotic analysis of the interfacial mode and showed that it may become unstable at vanish-

Correspondence concerning this paper should be addressed to T. C. Papanastasiou. ingly small Reynolds number in the presence of viscosity stratification. The analysis was extended by Yiantsios and Higgins (1988) and by Hooper (1989) to include the effects of an arbitrary wavelength, and by Anturkar et al. (1990) to include the effects of an additional third layer. The parameters controlling the stability of multilayer flow were:

- Viscosity ratios

- Thickness ratios or flow-rate ratios

- Density ratios

- Interfacial tensions of individual layers gravity

A linear stability analysis of two-layer plane Poiseuille flow of second-order liquids was carried out by Khan and Han (1977) using long-wavelength asymptotic analysis. They found that the onset of interfacial instability depends on both the viscosity and the elasticity ratios.

Comprehensive experimental studies of stability of a twolayer water/mineral oil system in a rectangular duct with an aspect ratio of 1:8 (height:width) were conducted by Kao and Park (1972). Based on linear stability analysis, Yiantsios and Higgins (1988) attributed the instability observed by Kao and Park to a shear mode rather than to an interfacial mode and concluded that the influence of the aspect ratio and the nonlinearity of the system need to be further investigated. Experiments were also performed by Han and Shetty (1978) with three- and five-layer polystyrene/low-density polyethylene 
systems. However the aspect ratio, the flow rates of the individual layers and some of the required rheological data of the polymer melts were not reported. Therefore, their results were inadequate to establish any criteria of instability.

Although existing work on multilayer plane Poiseuille flow is limited, a large number of studies on the stability of two- and three-layer Couette flows of Newtonian liquids have been carried out (Li, 1969a; Smith and Davis, 1982; Hooper and Boyd, 1983; Hooper, 1985; Renardy, 1987a; b; and others). Similarly, stability analysis of two-layer Couette flow of inelastic power-law liquids (Waters, 1983), of constant viscosity Oldroyd-B liquids ( $\mathrm{Li}, 1969 \mathrm{~b})$, and of Oldroyd liquids with shear-dependent viscosities (Waters and Keeley, 1987) were performed for long-wavelength disturbances. Also the stability of upper-convected Maxwell liquids (Renardy, 1988) was studied for disturbances of arbitrary wavelengths.

None of the works on plane Poiseuille flow considers the effects of shear thinning and viscoelasticity of polymer melts. Furthermore, most of the applications of multilayer films involve more than two layers. In this paper, a linear stability analysis of multilayer plane Poiseuille flow of Oldroyd-B liquids with shear rate dependent viscosities is performed. The primary goal is to investigate the effects of the shear rate dependent viscosity and the viscoelasticity of the individual layers on the stability of multilayer flow. The equations and the numerical algorithms are developed for $l$-layer fiow, where the number of layers $l$ is arbitrary. However, since large numbers of parameters are involved in the analysis, the results are presented only for two- and three-layer flows. Asymptotic solutions are constructed for two-dimensional disturbances of long wavelengths. Since the dominant mode of instability can be due to a disturbance of an arbitrary wavelength, the numerical solution to the corresponding eigenvalue problem is also obtained, and the neutral-stability curves are plotted over a wide spectrum of wavelengths. The objective is to construct operating diagrams defining the stable regions for all wavelengths in terms of the rheological parameters and the operating conditions.

\section{The Constitutive Equation}

In this investigation, a modified Oldroyd-B model is used that relates the stress tensor $\tau$ to the rate-of-strain tensor $\dot{\gamma}(=[\nabla v+(\nabla v) \dagger])$ :

$$
\tau+\lambda_{1} \tau_{(1)}=\eta(\dot{\gamma})\left[\dot{\gamma}+\lambda_{2} \dot{\gamma}_{(2)}\right]
$$

where the convected derivatives $\tau_{(1)}$ and $\gamma_{(2)}$ are given by

$$
\begin{gathered}
\boldsymbol{\tau}_{(1)}=\frac{\partial \tau}{\partial t}+\mathbf{v} \cdot \nabla \boldsymbol{\tau}-[\boldsymbol{\tau} \cdot \nabla \mathbf{v}+(\nabla \mathbf{v}) \dagger \cdot \tau] \\
\dot{\gamma}_{(2)}=\frac{\partial \dot{\gamma}}{\partial t}=\mathbf{v} \cdot \nabla \dot{\gamma}-[\dot{\gamma} \cdot \nabla \mathbf{v}+(\nabla \mathbf{v}) \dagger \cdot \dot{\gamma}]
\end{gathered}
$$

Here, $\lambda_{1}$ and $\lambda_{2}$ are the relaxation and retardation times respectively, and $\mathbf{v}$ is the velocity vector. The viscosity $\eta$ is a function of the magnitude $\dot{\gamma}\left(=[1 / 2 \dot{\gamma}: \dot{\gamma}]^{1 / 2}\right)$ of the rate-of-strain tensor and is given by the Carreau viscosity function (Bird et al., 1987):

$$
\eta(\dot{\gamma})=\eta_{0}\left(1+\lambda_{v}^{2} \dot{\gamma}^{2}\right)^{(n-1) / 2}
$$

where $\eta_{0}$ is the zero shear rate viscosity, $\lambda_{v}$ is the material time constant, and $n$ is the dimensionless power-law exponent. The model has five material constants. The constants $\eta_{0}, \lambda_{v}$, and $n$ can be determined from viscosity vs. shear rate data, and $\lambda_{1}$ and $\lambda_{2}$ can be determined from stress relaxation curves. At low shear rates, $\eta$ approaches the zero shear rate viscosity, whereas at high shear rates, it exhibits power-law behavior. Pressure-driven flows, such as the plane Poiseuille flow considered in this analysis, involve low shear rate regions within their domains. Therefore, the simple power-law model is not suitable for stability analyses of pressure-driven flows due to its prediction of infinite viscosity at zero shear rate. Stability results with the simple power-law model are expected to be particularly inaccurate when interfaces are located in low shear rate regions. In these cases, the Carreau viscosity function, incorporated here in the Oldroyd-B model, is an appropriate choice due to its prediction of finite viscosity at vanishingly small shear rates.

\section{Primary Flow}

The flow geometry is shown in Figure 1 together with a coordinate transformation that simplifies the governing equations. The dimensionless variables introduced are

$$
\begin{aligned}
& x^{*}=\frac{x}{H}, \quad y_{j}^{*}= \frac{y_{j}}{H}, \\
& \tau_{j}^{*}=\frac{\tau_{j}}{\left(\eta_{01} V / H\right)}, \quad p_{j}^{*}= \frac{p_{j}}{\left(\eta_{01} V / H\right)}, \\
& u_{j}^{*}=\frac{u_{j}}{V}, \quad v_{j}^{*}=\frac{v_{j}}{V}, \\
& t^{*}=\frac{t}{(H / V)}, \quad j=1,2, \ldots, \ell
\end{aligned}
$$

The subscript $j$ denotes the $j$ th layer, and the superscript asterisk, which is hereafter suppressed, denotes dimensionless variables. The variables $u_{j}$ and $v_{j}$ are the velocity components along the $x$ and $y_{j}$ directions respectively. $t$ is the time, $\tau_{j}$ the stress tensor, $p_{j}$ the pressure, and $l$ the total number of layers. The characteristic velocity $V$ is the total average velocity; the characteristic length $H$ is the total thickness of the channel; and the characteristic viscosity $\eta_{01}$ is the zero shear rate viscosity of the first layer. Henceforth, all equations are for $j=1,2, \ldots, l$, unless otherwise specified.

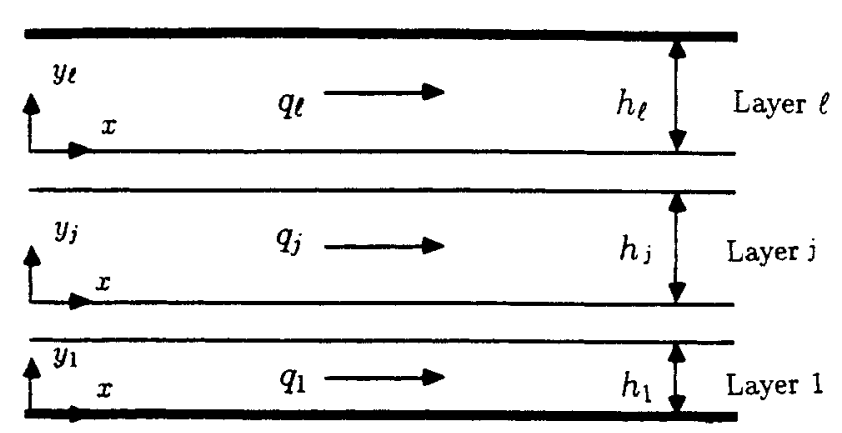

Figure 1. Multilayer plane Poiseuille flow and transformation of coordinates. 
The dimensionless equations of change are then expressed as:

$$
\begin{gathered}
\operatorname{Re}_{j}\left[\frac{\partial \mathbf{v}_{j}}{\partial t}+\mathbf{v}_{j} \cdot \nabla \mathbf{v}_{j}\right]= \\
\nabla \cdot \mathbf{v}_{j}=0
\end{gathered}
$$

where $R e_{j}=\left(\rho_{j} H V / \eta_{01}\right)=d_{j} R e_{1}$ is the Reynolds number, $d_{j}=$ $\rho_{j} / \rho_{1}$ is the density ratio, and $I$ is the unit tensor. The dimensionless constitutive equation becomes

$$
\tau_{j}+\Lambda_{1 j} \tau_{(1) j}=\Gamma_{j}\left(\dot{\gamma}_{j}\right)\left[\dot{\gamma}_{j}+\Lambda_{2 j} \dot{\gamma}_{(2) j}\right]
$$

in which $\Gamma_{j}\left(\dot{\gamma}_{j}\right)=\kappa_{j}\left[1+\Lambda_{v j}^{2} \dot{\gamma}_{j}^{2}\right]^{\left(n_{j-1}\right) / 2}$ is the dimensionless viscosity function, $\kappa_{j}=\eta_{0 j} / \eta_{01}$ is the zero shear rate viscosity ratio, and $\Lambda_{i j}=\lambda_{i j} \mathrm{~V} / \mathrm{H}$ (for $\lambda_{1 j}, \lambda_{2 j}$ and $\lambda_{v j}$ ) are the dimensionless time constants.

The primary flow is assumed to be fully developed onedimensional flow. Then the nonzero stresses, predicted by Eq. 8, are

$$
\tau_{x y j}=\Gamma_{j} \frac{d u_{j}}{d y_{j}}
$$

and

$$
\tau_{x x j}=2\left(\Lambda_{1 j}-\Lambda_{2 j}\right) \Gamma_{j}\left(\frac{d u_{j}}{d y_{j}}\right)^{2}
$$

For constant pressure gradients, the equations of motion reduce to

$$
\frac{d \tau_{x y j}}{d y_{j}}=\frac{d p_{j}}{d x}
$$

For flat interfaces, the pressure gradient is the same for all layers. Then from Eqs. 8 and 9,

$$
\Gamma_{j} \frac{d u_{j}}{d y_{j}}=C y_{j}+B_{j}
$$

where $C$ equals $d p_{j} / d x$ for all layers and $B_{j}$ is an integration constant.

The boundary conditions are

\section{No slip at the walls}

$$
u_{1}(0)=u_{l}\left(h_{l}\right)=0
$$

Continuity of velocity at the interfaces

$$
u_{j}\left(h_{j}\right)=u_{j+1}(0), j=1,2, \ldots, l-1
$$

\section{Continuity of shear stress at the interfaces}

$$
\tau_{x y j}\left(h_{j}\right)=\tau_{x y(j+1)}(0), \quad j=1,2, \ldots, l-1
$$

where $h_{j}$ is the thickness of the $j$ th layer.

The additional constraint introduced due to nondimensionalization of the variables is that the total dimensionless flow rate is equal to 1 , that is,

$$
\sum_{j=1}^{l} \int_{0}^{h_{j}} u_{j} d y_{j}=\sum_{j=1}^{l} q_{j}=1
$$

where $q_{j}$ is the flow rate per unit width, given that the summation of dimensionless thicknesses of all layers is equal to 1. Equations $12-14$ provide the solutions of $u_{j}, C$, and $B_{j}$ for a given set of thicknesses $h_{j}$.

Equations 12-13 can be solved numerically for a given initial guess of the pressure gradient by means of a pseudospectral method to discretize the variable $u_{j}\left(y_{j}\right)$ in each layer. The variable $u_{j}\left(y_{j}\right)$ is expanded in a series of Chebyshev polynomials $T_{k}(z)$, defined by $T_{k}(\cos \theta)=\cos (k \theta)$, and truncated. The details of the pseudospectral method are described elsewhere (Gottlieb and Orszag, 1977; Orszag, 1971). The resulting system of nonlinear algebraic equations is solved by Newton iteration. Once $u_{j}\left(y_{j}\right)$ and $B_{j}$ are determined for an assumed $C$, Eq. 14 is used to correct the value of $C$.

When $n_{j}=1$ or $\Lambda_{v j}=0$ for each layer, that is, when the viscosity of each layer is constant, Eq. 12 can be solved analytically for the parabolic velocity profiles. Then, Eqs. 13-14 form a set of nonlinear algebraic equations that can be solved simultaneously to estimate the pressure gradient, the velocity profiles, and the stresses.

\section{Secondary Flow}

Following Yih (1967), and Waters and Keeley (1987), two-dimensional infinitesimal disturbances are imposed on the above primary flow. The velocity components $u_{j}$ and $v_{j}$, the stress components $\tau_{x x j}, \tau_{x y j}$, and $\tau_{y y j}$, and the pressure $p$ are assumed to have the following general form,

$$
f_{j}\left(x, y_{j}, t\right)=f_{s j}\left(y_{j}\right)+\hat{f}_{j}\left(x, y_{j}, t\right)
$$

where $f_{s j}$ is the steady-state value and $\hat{f}_{j}$ is infinitesimally small. From the preceding section on primary flow, $v_{s j}=\tau_{y y s j}=0$. When Eq. 15 is substituted in the continuity equation, Eq. 7, the disturbances in velocity components can be expressed in terms of a stream function $\psi_{j}\left(x, y_{j}, t\right)$, where $\hat{u}_{j}=\partial \psi_{j} / \partial y_{j}$ and $\hat{v}_{\mathrm{j}}=$ $-\partial \psi_{j} / \partial x$. The disturbances are in the form of (Drazin and Reid, $1981)$

$$
\left(\hat{\psi}_{j}, \hat{p}, \hat{\tau}_{j}\right)=\left[\bar{\phi}_{j}\left(y_{j}\right), \bar{p}\left(y_{j}\right), \bar{\tau}_{j}\left(y_{j}\right)\right] \exp [i \alpha(x-c t)]
$$

where $\alpha$ is a real wave number, $c=c_{R}+i c_{T}$ is a complex velocity of disturbances, and quantities with overbars are amplitudes of the disturbances. When $c_{f}>0$, the equations are temporally unstable and when $c_{I}<0$, they are stable. Neutral stability corresponds to $c_{1}=0$. By substituting Eq. 16 in the linearized momentum equations and then eliminating the pressure, the resulting equations, for both Newtonian and viscoelastic liquids, are

$$
\begin{aligned}
i \alpha \operatorname{Re}_{j}\left(u_{s j}-c\right)\left(\phi_{j}^{\prime \prime}-\alpha^{2} \phi_{j}\right) & \\
& =\left(\tau_{x y j}^{\prime \prime}+\alpha^{2} \tau_{x y j}\right)+i \alpha\left(\tau_{x x j}^{\prime}-\tau_{y j j}^{\prime}\right)
\end{aligned}
$$

where the derivatives with respect to $y_{j}$ are denoted by primes, and overbars are suppressed hereafter.

The interfacial boundary conditions need to be evaluated at 
the disturbed locations of the interfaces. The Taylor series expansion of these interfacial boundary conditions about the steady-state interfacial locations and the no-slip boundary conditions at the walls give rise to the following set of boundary conditions.

No slip at the walls

$$
\begin{aligned}
& \phi_{1}(0)=\phi_{1}^{\prime}(0)=0 \\
& \phi_{l}\left(h_{l}\right)=\phi_{l}^{\prime}\left(h_{l}\right)=0
\end{aligned}
$$

Continuity of $u_{j}$ at the interfaces

$$
\phi_{j}^{\prime}+\frac{\phi_{j} u_{s j}^{\prime}}{\tilde{c}_{j}}=\phi_{j+1}^{\prime}+\frac{\phi_{j+1} u_{s(j+1)}^{\prime}}{\tilde{c}_{j+1}}
$$

Continuity of $v$, at the interfaces

$$
\phi_{j}=\phi_{j+1}
$$

Continuity of shear stress at the interfaces

$$
\tau_{x y j}=\tau_{x y(j+1)}
$$

Continuity of normal stress at the interfaces

$$
\begin{aligned}
& {\left[\tau_{x y(j+1)}^{\prime}+i \alpha\left(\tau_{x x(j+1)}-\tau_{y y(j+1)}\right)\right.} \\
& \left.+\operatorname{Re}_{j+1}\left(i \alpha \phi_{j+1}^{\prime} \tilde{c}_{j+1}+i \alpha u_{s(j+1)}^{\prime} \phi_{j+1}\right)\right] \\
& -\left[\tau_{x y j}^{\prime}+i \alpha\left(\tau_{x x j}-\tau_{y y j}\right)+\operatorname{Re}_{j}\left(i \alpha \phi_{j}^{\prime} \tilde{c}_{j}+i \alpha u_{s j}^{\prime} \phi_{j}\right)\right] \\
& =\left[i \alpha^{3} / C a_{j}+i \alpha S t\left(d_{j+1}-d_{j}\right)\right] \frac{\phi_{j}}{\tilde{c}_{j}}
\end{aligned}
$$

Here $\tilde{c}_{j}=c-u_{s j}$, and the dimensionless groups $S t=\left(H^{2} \mathrm{~g} \rho_{1}\right) /$ $\left(V \eta_{01}\right)$ and $C a_{j}=V \eta_{01} / \sigma_{j}$ are the Stokes number (proportional to gravity $g$ ) and the capillary number (inversely proportional to interfacial tension $\sigma_{j}$ ), respectively. In Eqs. 18c-18f, the variables with subscript $j$ are evaluated at $y_{j}=h_{j}$ and the variables with subscript $j+1$ are evaluated at $y_{j+1}=0$, where $j=$ $1,2, \ldots, l-1$.

Equations 17-18 involve the perturbed stress components. By substituting Eq. 16 into the constitutive equation, Eq. 8, and retaining only the linear terms of the perturbed quantities, the expressions for the stress components are

$$
\begin{array}{r}
\left.\tau_{y y j}=\left\{2 \alpha^{2} \Lambda_{1 j} \tau_{x y s j} \phi_{j}-\Gamma_{j}\left(u_{s j}^{\prime}\right)\left[2 i \alpha \phi_{j}^{\prime}+2 \alpha^{2} \Lambda_{2 j}\left(\phi_{j}^{\prime} \tilde{c}_{j}+u_{s j}^{\prime} \phi_{j}\right)\right]\right\}\right\} \\
\left(1-i \alpha \Lambda_{1 j} \tilde{c}_{j}\right) \quad(19 \mathrm{a})
\end{array}
$$

$$
\begin{gathered}
\tau_{x y j}=\left(\Lambda_{1 j}\left(i \alpha \tau_{x y s j}^{\prime} \phi_{j}+\alpha^{2} \tau_{x x s j} \phi_{j}+u_{s j}^{\prime} \tau_{y y j}\right)\right. \\
+\Gamma_{j}\left(u_{s j}^{\prime}\right)\left\{\tilde{n}_{j}\left(\phi_{j}^{\prime \prime}+\alpha^{2} \phi_{j}\right)\right. \\
+i \alpha \Lambda_{2 j}\left[2 u_{s j}^{\prime} \phi_{j}^{\prime}-u_{s j}^{\prime \prime} \phi_{j}-\tilde{c}_{j}\left(\phi_{j}^{\prime \prime}\right.\right. \\
\left.\left.\left.\left.+\alpha^{2} \phi_{j}\right)\right]\right\}\right) /\left(1-i \alpha \Lambda_{1 j} \tilde{c}_{j}\right) \\
\tau_{x x j}=\left(\Lambda_{1 j}\left(i \alpha \tau_{x x s j}^{\prime} \phi_{j}+2 i \alpha \tau_{x x s j} \phi_{j}+2 \tau_{x y s j} \phi_{j}^{\prime \prime}+2 \tau_{x y j} u_{s j}^{\prime}\right)\right. \\
+\Gamma_{j}\left(u_{s j}^{\prime}\right)\left\{2 i \alpha \phi_{j}^{\prime}+\Lambda_{2 j}\left[2 \alpha^{2} \tilde{c}_{j} \phi_{j}^{\prime}\right.\right. \\
\left.\left.\left.-2\left(\tilde{n}_{j}+1\right) u_{s j}^{\prime} \phi_{j}^{\prime \prime}-2 \tilde{n}_{j} \alpha^{2} u_{s j}^{\prime} \phi_{j}\right]\right\}\right) /\left(1-i \alpha \Lambda_{l j} \tilde{c}_{j}\right),
\end{gathered}
$$

where

$$
\widetilde{n}_{j}=1+\frac{\left(n_{j}-1\right) \Lambda_{v j}^{2} u_{s j}^{\prime 2}}{1+\Lambda_{v j}^{2} u_{s j}^{\prime 2}}
$$

After some algebraic manipulations of Eq. 19, these stress components can be expressed explicitly in terms of $\phi_{j}\left(y_{j}\right)$ and its derivatives.

Equations 17-19 form an eigenvalue problem with the complex velocity of disturbance $c$ as the eigenvalue and the amplitudes of disturbance $\phi_{j}\left(y_{j}\right)$ as the eigenfunctions. The eigenvalue depends on the parameters $m_{j}, q_{j}$ or $h_{j}, d_{j}, R e_{j}, \Lambda_{l j}, \Lambda_{2 j}, n_{j}, \Lambda_{v j}$, and $C a_{j}$ of all the layers; and on $\alpha$ and $S t$. The large number of parameters introduced is due to the presence of the interfaces. Therefore, although the further analysis and algorithms are developed for $l$-layer flow, the results are presented only for twoand three-layer flows. To restrict further the number of involved parameters, the interfacial tensions and gravity are assumed to be negligible compared to viscous forces, which is reasonable for polymer melts due to their high viscosities. Also, the retardation time $\Lambda_{2 j}$ is assumed to be equal to $\Lambda_{1 j} / 9$, a commonly observed value for the Oldroyd-B model. All the results are presented in terms of flow rates $q_{j}$, rather than thicknesses $h_{j}$, because the flow rates are the controlling parameters in industrial operations involving multilayer extrusion.

\section{Asymptotic Analysis $\left(\alpha \rightarrow 0, n_{J}=1\right)$}

Prior to the numerical analysis of the above eigenvalue problem, an asymptotic analysis is carried out for longwavelength disturbances $(\alpha \rightarrow 0)$. This asymptotic analysis gives rise to algebraic equations and saves computational time. In addition, the numerical algorithm developed for an arbitrary $\alpha$ can be tested at small $\alpha$ by comparing the results of the asymptotic analysis with the numerical predictions. Furthermore, in many cases asymptotic analysis may provide the critical stability parameters, as discussed in the next section.

Here, the asymptotic analysis is restricted to constantviscosity liquids in each of the layers $\left(n_{j}=1\right)$. The eigenfunctions and the eigenvalues are expanded as regular perturbation series of $\alpha$ :

$$
\begin{aligned}
\phi_{j} & \simeq \phi_{j, 0}+\alpha \phi_{j, 1}+O\left(\alpha^{2}\right) \\
c & \simeq c_{0}+\alpha c_{1}+O\left(\alpha^{2}\right)
\end{aligned}
$$

where the subscripts 0 and 1 denote the zero- and the first-order terms, respectively. Equation 20 is substituted in Eqs. 17-19 and terms of the same order of $\alpha$ are collected. The resulting equations are solved sequentially for the coefficients of $\phi_{j}$ and $c$ at each order. Since the procedure is standard, only the final form of the equations is listed here.

The zero-order solution for the eigenfunctions is

$$
\phi_{j, 0}=D_{j, 0}+E_{j, 0} y_{j}+F_{j, 0} y_{j}^{2}+G_{j, 0} y_{j}^{3} \quad 0 \leq y_{j} \leq h_{j}
$$

where, $D_{j, 0}, E_{j, 0}, F_{j, 0}$, and $G_{j, 0}$ are constants. By substituting Eq. 21 in the zero-order boundary conditions, an eigenvalue problem with $4 l$ algebraic equations is obtained. The relaxation and retardation times do not enter in the zero-order terms. There- 
fore, the zero-order equations are identical to those for Newtonian liquids, described by Anturkar et al. (1990). The solution of these equations is real, implying that $c_{0 t}=0$.

The first-order solution of $\phi_{j}\left(y_{j}\right)$ is

$$
\begin{aligned}
\phi_{j, 1}=D_{j, 1}+E_{j, 1} y_{j}+F_{j, 1} y_{j}^{2}+G_{j, 1} y_{j}^{3}+\chi_{j}\left(y_{j}\right) & \\
& 0 \leq y_{j} \leq h_{j}
\end{aligned}
$$

with

$$
\begin{aligned}
\chi_{j}\left(y_{j}\right)= & \frac{U_{j, 0}}{24} y_{j}^{4}+\frac{U_{j, 1}}{120} y_{j}^{5}+\frac{U_{j, 2}}{360} y_{j}^{6}+\frac{U_{j, 3}}{840} y_{j}^{7} \\
U_{j, 0}= & \frac{2 i R e_{j}}{\kappa_{j}}\left[-c_{0} F_{j, 0}+A_{j} F_{j, 0}-\left(C / 2 \kappa_{j}\right) D_{j, 0}\right] \\
U_{j, 1}= & \frac{2 i R e_{j}}{\kappa_{j}}\left[-3 c_{0} G_{j, 0}\right. \\
& \left.+3 A G_{j, 0}+\left(B_{j} / \kappa_{j}\right) F_{j, 0}-\left(C / 2 \kappa_{j}\right) E_{j, 0}\right] \\
U_{j, 2}= & \frac{6 i R e_{j} B_{j} G_{j, 0}}{\kappa_{j}^{2}} \\
U_{j, 3}= & \frac{2 i R e_{j} C G_{j, 0}}{\kappa_{j}^{2}}
\end{aligned}
$$

where $D_{j}, E_{j, 1}, F_{j, 1}$, and $G_{j, 1}$ are constants and $A_{j}$ and $B_{j}$ are the constant coefficients of the steady-state parabolic velocity profiles. By substituting Eq. 22 in the first-order boundary condition, $4 l$ algebraic equations are obtained. They are:

$$
\begin{aligned}
& D_{1,1}=0 \\
& E_{1,1}=0
\end{aligned}
$$

$$
D_{j, 1}+E_{j, 1} h_{j}+F_{j, 1} h_{j}^{2}+G_{j, 1} h_{j}^{3}+\chi_{j}\left(h_{j}\right)-D_{j+1,1}=0
$$

$$
\begin{aligned}
\tilde{c}_{j, 0}\left(h_{j}\right)\left[E_{j, 1}+2 F_{j, 1} h_{j}+3 G_{j, 1} h_{j}^{2}+\chi_{j}^{\prime}\left(h_{j}\right)\right] \\
+u_{s j}^{\prime}\left(h_{j}\right)\left[D_{j, 1}+E_{j, 1} h_{j}+F_{j, 1} h_{j}^{2}+G_{j, 1} h_{j}^{3}+\chi_{j}\left(h_{j}\right)\right] \\
+c_{1}\left[E_{j, 0}+2 F_{j, 0} h_{j}+3 G_{j, 0} h_{j}^{2}\right]-\tilde{c}_{j+1,0}(0) E_{j+1,1} \\
\quad-D_{j+1,1} B_{j+1} / \kappa_{j+1}-c_{1} E_{j+1,0}=0
\end{aligned}
$$

$$
\begin{gathered}
\kappa_{j}\left[2 F_{j, 1}+6 G_{j, 1} h_{j}+\chi_{j}^{\prime \prime}\left(h_{j}\right)\right]-2 \kappa_{j+1} F_{j+1,1}+i \kappa_{j}\left(\Lambda_{1 j}-\Lambda_{2 j}\right) \\
{\left[\frac{C}{\kappa_{j}}\left(D_{j, 0}-E_{j, 0} h_{j}-3 F_{j, 0} h_{j}^{2}-5 G_{j, 0} h_{j}^{3}\right)\right.} \\
\left.\quad-2 \frac{B_{j}}{\kappa_{j}}\left(E_{j, 0}+2 F_{j, 0} h_{j}+3 G_{j, 0} h_{j}^{2}\right)\right] \\
+i \kappa_{j}\left(\Lambda_{1 j}-\Lambda_{2 j}\right) \tilde{c}_{j, 0}\left(h_{j}\right)\left(2 F_{j, 0}+6 G_{j, 0} h_{j}\right) \\
-i \kappa_{j+1}\left[\Lambda_{l(j+1)}-\Lambda_{2(j+1)}\right]\left[\frac{C}{\kappa_{j+1}} D_{j+1,0}\right. \\
\left.\quad-2 \frac{B_{j+1}}{\kappa_{j+1}} E_{j+1,0}+2 \tilde{c}_{j+1}(0) F_{j+1,0}\right]=0
\end{gathered}
$$

$$
\begin{gathered}
6 \kappa_{j+1} G_{j+1,1}-6 \kappa_{j} G_{j, 1}-\kappa_{j} \chi_{j}^{\prime \prime \prime}\left(h_{j}\right)+i R e_{j+1} \\
{\left[\tilde{c}_{j+1,0}(0) E_{j+1,0}+D_{j+1,0} \frac{B_{j+1}}{\kappa_{j+1}}\right]} \\
-i R e_{j} \tilde{c}_{j, 0}\left(h_{j}\right)\left(E_{j, 0}+2 F_{j, 0} h_{j}+3 G_{j, 0} h_{j}^{2}\right) \\
-i R e_{j} u_{s j}^{\prime}\left(h_{j}\right)\left(D_{j, 0}+E_{j, 0} h_{j}+F_{j, 0} h_{j}^{2}+G_{j, 0} h_{j}^{3}\right) \\
+i \kappa_{j+1}\left[\Lambda_{l(j+1)}-\Lambda_{2(j+1)}\right]\left[2 \frac{B_{j+1}}{\kappa_{j+1}} F_{j+1,0}\right. \\
\left.-\frac{C}{\kappa_{j+1}} E_{j+1,0}+6 \tilde{c}_{j+1}(0) G_{j+1,0}\right] \\
-i \kappa_{j}\left(\Lambda_{l j}-\Lambda_{2 j}\right)\left[\frac{C}{\kappa_{j}}\left(3 G_{j, 0} h_{j}^{2}-E_{j, 0}\right)\right. \\
\left.+\frac{B_{j}}{\kappa_{j}}\left(2 F_{j, 0}+6 G_{j, 0} h_{j}\right)+6 \tilde{c}_{j, 0}\left(h_{j}\right) G_{j, 0}\right]=0 \\
+E_{l, 1} h_{l}+F_{l, 1} h_{l}^{2}+G_{l, 1} h_{l}^{3}+\chi_{l}\left(h_{l}\right)=0 \\
E_{l, 1}+2 F_{l, 1} h_{l}+3 G_{l, 1} h_{l}^{2}+\chi_{l}^{\prime}\left(h_{l}\right)=0
\end{gathered}
$$

where $j=1,2, \ldots, l-1$. The terms involving the relaxation and retardation times enter only in the form of $\left(\Lambda_{1 j}-\Lambda_{2 j}\right)$ in the boundary conditions of continuity of shear and normal stresses at the interfaces. Equations 22-23 constitute an eigenvalue problem with the first-order eigenvalue $c_{1}$.

From Eqs. 22-23, $c_{1}=i \operatorname{Re}_{1} f\left[c_{0}, d_{j}, \kappa_{j}, h_{j},\left(\Lambda_{1 j}-\Lambda_{2 j}\right)\right]$. Since the zero-order solutions is real, the first-order solution is purely imaginary. Therefore, the eigenvalue $c_{1}$ determines the stable and the unstable conditions. Since $c_{1} \propto R e_{1}$, a nonzero Reynolds number is essential for unstable flow to exist. However, the neutral-stability curves do not depend on the Reynolds numbers. It can be shown from algebraic manipulations of Eqs. 22-23 for two-layer flow that the neutral-stability curves of viscoelastic liquids include those of Newtonian liquids. Any other neutralstability curves for viscoelastic liquids need to be determined by the numerical solution of Eqs. 22-23.

The numerical solutions show that although the eigenvalues of the viscoelastic liquids differ from those of Newtonian liquids, the zeros of these eigenvalues of both liquids coincide in the case of two-layer flow. Therefore, there are no additional neutralstability curves of viscoelastic liquids compared to those of Newtonian liquids. In other words, the neutral-stability diagrams for $\alpha \rightarrow 0$ are identical for Newtonian and viscoelastic liquids in two-layer flow. As pointed out by Yih (1967), Yiantsios and Higgins (1988), and by Hooper (1989), the viscosity stratification is primarily responsible for the interfacial instability at long wavelengths. The neutrally stable flow occurs exactly at $q_{2} / q_{1}=h_{2} / h_{1}=\sqrt{\kappa_{2}}$, where the slope of the base velocity is zero, and hence continuous across the interface. Therefore, the neutral-stability diagrams are expected to be identical for Newtonian and viscoelastic liquids in the channel flow, where elasticity does not play any role in determining the primary velocity profiles. Besides, the period of oscillation for the fluid particles following the path of flow with disturbance of very large wavelengths is much greater than the relaxation time of the fluids. Therefore, the disturbances of very large wavelengths will induce identical behavior between Newtonian and 
viscoelaştic liquids. Since the neutral stability curves for Newtonian liquids are reported elsewhere (Yih, 1967; Yiantsios and Higgins, 1988), they are omitted here.

For three-layer flow, however, apart from the neutral-stability curves identical to those for Newtonian liquids, additional neutral-stability curves exist purely due to the viscoelastic nature of the liquids. At high elasticity these neutral-stability curves exist at $q_{3} / q_{1}$ close to 1.0. One such neutral-stability diagram is plotted in Figure 2 for $q_{3} / q_{1}=1.0,0.9$, and 0.8 in the plane of the relaxation time of the first layer $\Lambda_{11}$ and the flow-rate ratio $q_{2} / q_{1}$. The other parameters are $d_{j}=1, R e_{1}=$ $0.1, \kappa_{2}=5.0, \kappa_{3}=1.0, \Lambda_{12} / \Lambda_{11}=0.1, \Lambda_{13} / \Lambda_{11}=1.0$, and $\Lambda_{2 j}=$ $\Lambda_{1 j} / 9$. There are two neutral-stability curves for each $q_{3} / q_{1}$. The neutral-stability curve of constant $q_{2} / q_{1}$, denoted by $\left(q_{2} / q_{1}\right)_{c}$, does not depend of $\Lambda_{11}$ and is identical to that of Newtonian liquids. With increasing $q_{3} / q_{1},\left(q_{2} / q_{1}\right)_{c}$ decreases and at $q_{3} / q_{1}=1.0,\left(q_{2} / q_{1}\right)_{c}=0.0$. The other neutral-stability curve depends on $\Lambda_{11}$ and shrinks dramatically as $q_{3} / q_{1}$ departs from 1.0. The smallest $\Lambda_{11},\left(\Lambda_{11}\right)_{s}$, at which this unstable region occurs increases with decreasing $q_{3} / q_{1}$ as shown in Figure 2 . Since by interchanging layers 1 with 3 the same results are obtained, the results at $q_{3} / q_{1}>1.0$ are not shown in Figure 2. Our calculations also suggest that with increasing $\kappa_{2}$ similar results are obtained at lower $\left(\Lambda_{11}\right)_{s}$. These neutral-stability curves also exist at $\kappa_{3}<1$ or at $\kappa_{3}>1$. However, $\left(\Lambda_{11}\right)_{s}$ increases dramatically as $\kappa_{3}$ departs from 1 . Thus, symmetric configurations with more viscous liquid in the core layer are more unstable at high elasticity, whereas symmetric configurations with low-viscosity liquid in the core layer are more stable at high elasticity for long-wave perturbations. Results similar to those in Figure 2 are also obtained at high $\Lambda_{12}$ and / or at high $\Lambda_{13}$.

Note that the viscosity stratification is responsible for the onset of interfacial instability in three-layer flow as well. However, the continuity of the slope of the primary velocity profiles can exist only at one of the two interfaces. The other interface can still become stable or unstable, depending on the operating and rheological parameters. Therefore, the correla-

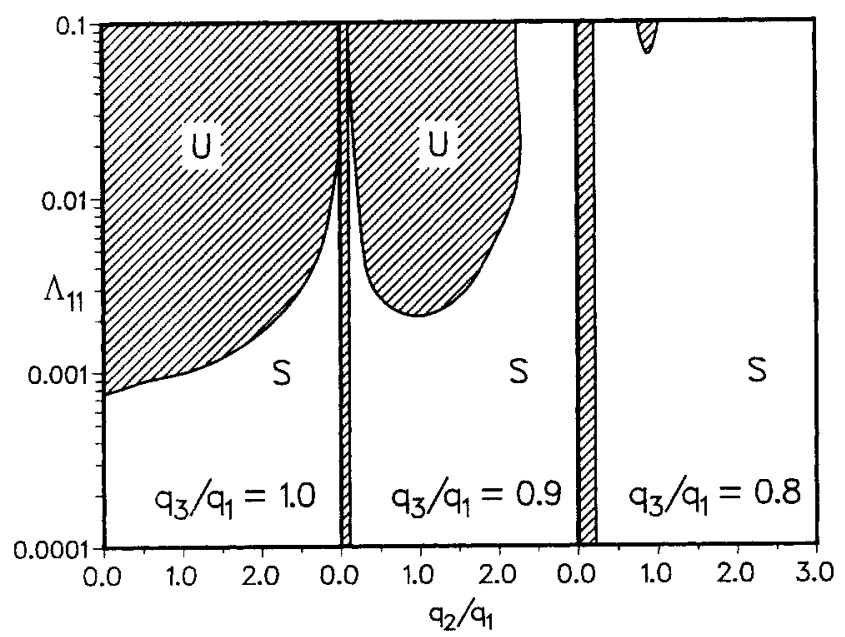

Figure 2. Neutral-stability curves for three-layer flow in $\Lambda_{11}-q_{2} / q_{1}$ plane at $\alpha \rightarrow 0$ for various flow rate ratios $q_{3} / q_{1}$.

Parameters: $\Lambda_{2 j} / \Lambda_{1 j}=1 / 9 ; \Lambda_{12} / \Lambda_{11}=0.1 ; d_{j}=1 ; \kappa_{2}=5.0 ; \kappa_{3}=1.0$ Hatched regions are unstable, unhatched regions are stable tion of neutrally stable conditions, similar to two-layer flow, does not exist for three-layer flow of either Newtonian or viscoelastic liquids. The existence of additional unstable regions in Figure 2 can be due to a resonance that prevails when a free wave at one interace forces a free wave at the other interface. This resonance phenomenon can occur only in the presence of at least two interfaces and has been observed by Taylor (1931) for inviscid fluids, and by $\mathrm{Li}$ (1969a) in the analysis of three-layer Couette flow. The neutral-stability curves of three-layer flow that are identical to those of Newtonian liquids are reported in Anturkar et al. (1990) and are not repeated here.

\section{Numerical Analysis}

The asymptotic analysis is valid at $\alpha \rightarrow 0$. However, the dominant mode of instability can be of any arbitrary wavelength. Therefore, the eigenvalue problem in Eqs. 17-19 was solved numerically for arbitrary $\alpha$. Since the perturbed stress components can be expressed explicitly in terms of $\phi_{j}\left(y_{j}\right)$, the stress components are eliminated from Eqs. $17-18$ by using Eq. 19. Thus, the only variable in the numerical analysis is $\phi_{j}\left(y_{j}\right)$. Similar to the numerical analysis of the primary flow, the pseudospectral method is chosen to discretize the eigenfunction $\phi_{j}\left(y_{j}\right)$ in each layer. If $N$ is the highest degree of the Chebyshev polynomial used to represent $\phi_{j}\left(y_{j}\right)$, the polynomials contribute $N+1$ unknowns in each layer, and Eq. 17 is represented at $N-$ 3 collocation points. Then along with the boundary conditions in Eq. 18, a matrix of order $l(N+1)$ is obtained. If the stress components are not expressed explicitly in terms of $\phi_{j}\left(y_{j}\right)$ and are treated as variables in the numerical analysis, then the resulting matrix is of order $4 l(N+1)$, which increases the computational cost substantially. The calculations were performed on an IBM 3090/600E mainframe computer with double-precision arithmetic. In almost all the cases, the relative change in the eigenvalues was less than $10^{-6}$ for $N>20$ in the case of constant-viscosity liquids and for $N>50$ in the case of shear rate dependent viscosity liquids.

The dominant eigenvalue of the matrix was tracked by using a shooting technique. The asymptotic solutions were used for predicting the initial guess of the eigenvalue at small $\alpha$. The initial guesses in the domain of the parameter space were then obtained by first-order continuation. Tracking of the dominant eigenvalue was ensured in several cases by computing all the eigenvalues of the matrix with the EISPACK routine. Additional caution was required in tracking the dominant eigenvalue by the shooting technique in three-layer flow. Corresponding to each interface, there is one eigenvalue that can become unstable at very small Reynolds number. In three-layer flow, there are two such eigenvalues that are close to each other, as shown in Table 1. Therefore, there exists a risk of tracking the eigenvalue close to the dominant eigenvalue, instead of the actual dominant eigenvalue itself, and thus obtaining inaccurate neutral-stability curves.

The eigenvalues computed by the pseudospectral method were compared with the eigenvalues computed by the compound matrix method (Anturkar et al., 1990) for multilayer flow of Newtonian liquids. The relative difference was less than $10^{-3}$. The asymptotic solutions at $\alpha \rightarrow 0$ are compared with the numerical results at small $\alpha$ in Table 2 for some representative parameters. The wave speed calculated by these two methods agree well for $\alpha=0.001$, and, as expected, deviate marginally 
Table 1. Two Close Eigenvalues That Can Become Unstable in Three-Layer Flow

\begin{tabular}{cll}
\hline$\alpha$ & & $q_{2} / q_{1}=0.8$ \\
\hline 0.1 & $1.3851127-0.2079156 \times 10^{-6} i$ & $1.2699918-0.1937410 \times 10^{-4} i$ \\
0.5 & $1.3867540-0.7972569 \times 10^{-6} i$ & $1.2723974-0.9515586 \times 10^{-4} i$ \\
1.0 & $1.3914035-0.3293587 \times 10^{-6} i$ & $1.2801425-0.1792529 \times 10^{-3} i$ \\
5.0 & $1.4334191+0.1461815 \times 10^{-4} i$ & $1.4564182+0.8733451 \times 10^{-4} i$ \\
10.0 & $1.4456493+0.5538804 \times 10^{-5} i$ & $1.4623606+0.3772467 \times 10^{-4} i$ \\
15.0 & $1.4489230-0.1835139 \times 10^{-6} i$ & $1.4546264-0.4721042 \times 10^{-5} i$ \\
\hline & & \\
\hline 0.1 & & $q_{2} / q_{1}=1.5$ \\
1.0 & $1.2638043-0.5428531 \times 10^{-6} i$ & $1.1543518-0.2193862 \times 10^{-4} i$ \\
5.0 & $1.2668594-0.2385817 \times 10^{-5} i$ & $1.1567993-0.1085625 \times 10^{-3} i$ \\
10.0 & $1.2756070-0.2994051 \times 10^{-5} i$ & $1.1649184-0.2094305 \times 10^{-3} i$ \\
15.0 & $1.3613317+0.1918564 \times 10^{-4} i$ & $1.3898547+0.6456265 \times 10^{-5} i$ \\
\hline
\end{tabular}

Parameters: $\Lambda_{11}=0.0005 ; \Lambda_{12} / \Lambda_{11}=0.1 ; \Lambda_{13} / \Lambda_{11}=1.0 ; R e_{j}=0.1 ; d_{j}=1, \kappa_{2}=5.0, \kappa_{3}=1.0$

as $\alpha$ increases from 0.001 to 0.1 . A consistency test described by Renardy (1987b) was also performed. In this test, when liquids 1 and 2 have identical properties in three-layer flow, one interfacial eigenvalue should be neutrally stable and the other should be independent of $q_{2} / q_{1}$. The same test was also performed when liquids 2 and 3 have identical properties. An additional check was performed by interchanging layers 1 and 2 in two-layer flow, and layers 1 and 3 in three-layer flow, where as expected, the eigenvalues and the neutral-stability diagrams did not chan $z$ in the absence of gravity. A representative example of a neutral-stability diagram of two-layer flow in the plane of $\alpha-q_{2} / q_{1}$ is shown in Figure 3. All the material properties in Figure $3 \mathrm{a}$ are inverted in Figure $3 \mathrm{~b}$ by interchanging the layers. The neutral-stability diagrams in Figure $3 \mathrm{a}$ and $3 \mathrm{~b}$ are essentially identical. They differ in shape only because Figure $3 \mathrm{~b}$ is plotted in the $\alpha-q_{2} / q_{1}$ plane, rather than in the $\alpha-q_{1} / q_{2}$ plane.

Due to the presence of multiple interfaces and due to the viscoelastic and the shear-thinning behavior of the liquids, a large number of parameters that affect the stability of the multilayer flow are involved in the analysis. Therefore, it is impossible to examine the effect of each parameter over the complete spectrum of all other parameters. In the numerical results presented below, we have chosen the cases that highlight the qualitative effects of the parameters under investigation, namely the relaxation times, the viscosity ratios, and the flow-rate ratios. The generalizations drawn here are expected to characterize the stability of similar multilayer flows in industrial processes.

\section{Liquids with constant viscosity}

The effect of the relaxation time of the first layer $\Lambda_{11}$ on the neutral-stability curves of two-layer flow of liquids with constant viscosity $\left(n_{j}=1\right)$ is shown in Figure 4 in the plane of the wave number $\alpha$ and the flow-rate ratio $q_{2} / q_{1}$. The liquid in the second layer is more viscous $\left(\kappa_{2}=5.0\right)$ and the ratio of the relaxation times $\Lambda_{12} / \Lambda_{11}$ is equal to 0.1 . As mentioned earlier, the retardation time $\Lambda_{2 j}$ is equal to $\Lambda_{1 j} / 9$ in all the calculations. The Reynolds number $R e_{1}$ is equal to 0.1 and the density ratio $d_{j}$ equals 1 . For each $\Lambda_{11}$, there are two neutral-stability curves.

Table 2. Comparison of Asymptotic and Numerical Solutions of Two-layer Flow

\begin{tabular}{|c|c|c|c|}
\hline$\alpha$ & \multicolumn{3}{|c|}{$q_{1} / q_{2}$} \\
\hline & & $\Lambda_{11}=0.10$ & \\
\hline- & $1.393353+0.2862605 \times 10^{-1} \alpha i$ & Asymptotic & $1.634750+0.5575722 \times 10^{-2} \alpha i$ \\
\hline $\begin{array}{l}0.001 \\
0.01 \\
0.1\end{array}$ & $\begin{array}{l}1.393353+0.2862602 \times 10^{-4} i \\
1.393331+0.2862282 \times 10^{-3} i \\
1.393128+0.2861346 \times 10^{-2} i\end{array}$ & Numerical & $\begin{array}{l}1.634743+0.5575719 \times 10^{-5} i \\
1.634726+0.5575546 \times 10^{-4} i \\
1.634189+0.5562357 \times 10^{-3} i\end{array}$ \\
\hline & & $\Lambda_{11}=0.0003$ & \\
\hline- & $1.393353+0.2986057 \times 10^{-3} \alpha i$ & Asymptotic & $1.634750+0.1144833 \times 1-^{-3} \alpha i$ \\
\hline $\begin{array}{l}0.001 \\
0.01 \\
0.1\end{array}$ & $\begin{array}{l}1.393353+0.2986051 \times 10^{-6} i \\
1.393334+0.2985050 \times 10^{-5} i \\
1.393035+0.2982568 \times 10^{-4} i\end{array}$ & Numerical & $\begin{array}{l}1.634745+0.1144830 \times 10^{-6} i \\
1.634623+0.1144356 \times 10^{-5} i \\
1.634282+0.1143200 \times 10^{-4} i\end{array}$ \\
\hline
\end{tabular}

Parameters: $R e_{j}=0.1 ; d_{j}=1 ; \Lambda_{12} / \Lambda_{1\}}=0.1 ; \kappa_{2}=5.0$ 


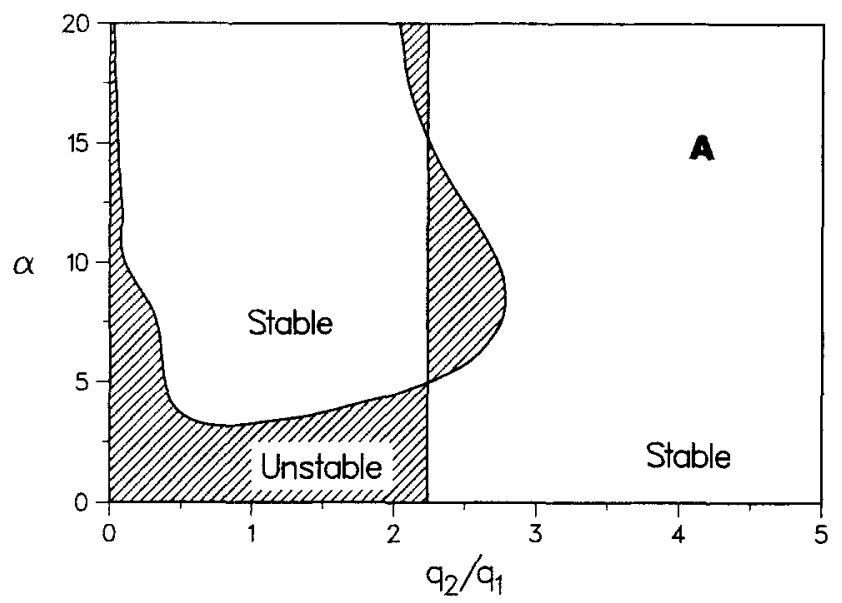

A. Parameters: $R e_{1}=0.1 ; \kappa_{2}=5.0 ; \Lambda_{12} / \Lambda_{11}=0.1 ; \Lambda_{11}=0.0005$

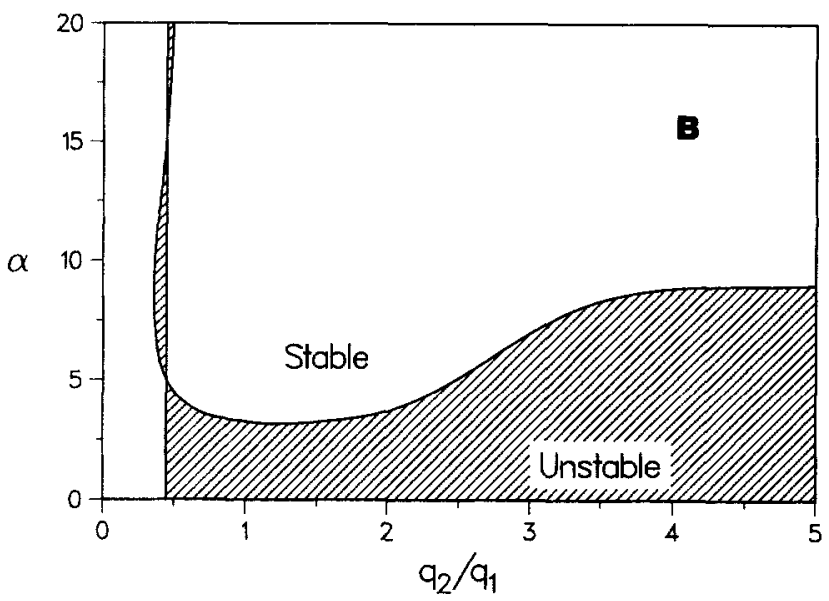

B. Curves plotted by interchanging layers in (a)

Figure 3. Neutral-stability curves for two-layer flow.

Parameters: $R e_{1}=0.2 ; \kappa_{2}=0.2 ; \Lambda_{12} / \Lambda_{11}=10.0 ; \Lambda_{11}=5.0 \times 10^{-5}$ Hatched regions are unstable, unhatched regions are stable

The neutral-stability curve of constant $q_{2} / q_{1}$, denoted by $\left(q_{2} /\right.$ $\left.q_{1}\right)_{s}$, corresponds to the asymptotic solution at $\alpha \rightarrow 0$ and is the same for all $\Lambda_{! !}$. As discussed previously, $\left(q_{2} / q_{1}\right)_{c}=h_{2} / h_{1}=$ $\sqrt{\kappa_{2}}$, and the slope of the base velocity profile is continuous across the interface under these conditions. For Newtonian liquids, Yiantsios and Higgins (1988) and Hopper (1989) found
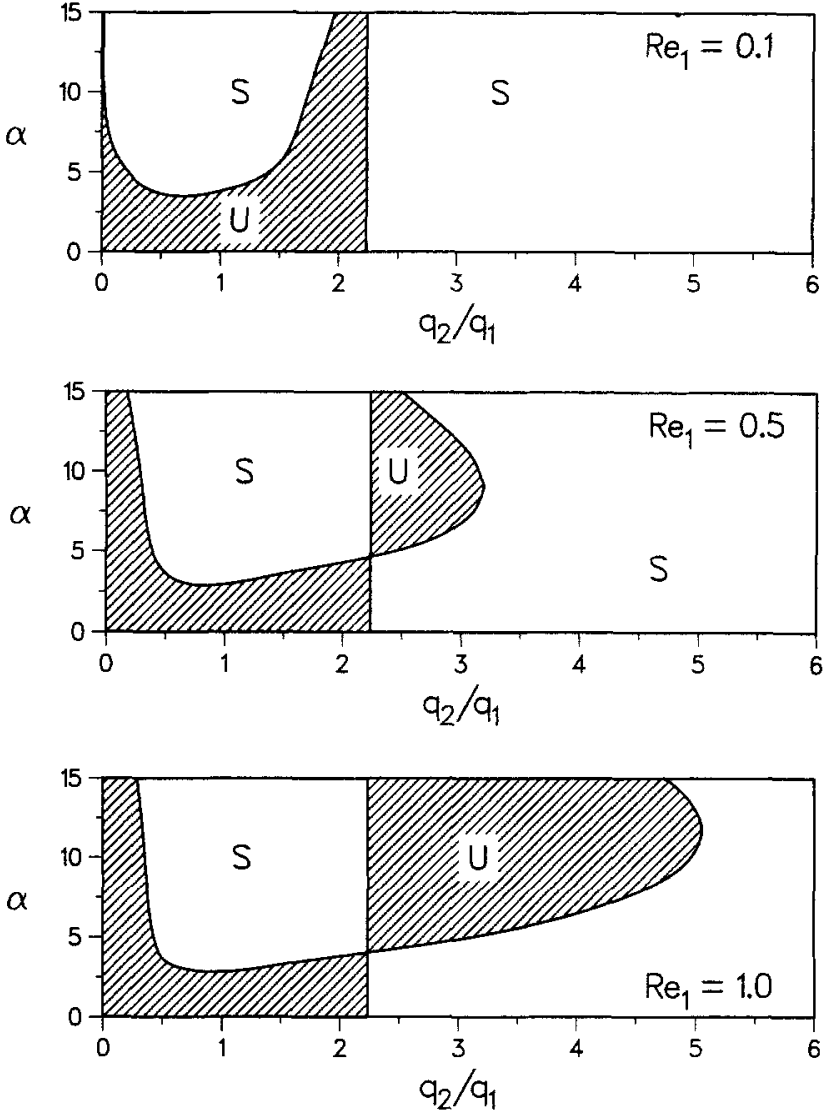

Figure 5. Neutral-stability curves for two-layer flow at various $R e_{1}$.

Parameters: $\Lambda_{11}=0.002 ; d_{i}=1 ; \kappa_{2}-5.0 ; \Lambda_{12} / \Lambda_{14}=0.1$

Hatched regions are unstable, unhatched regions are stable

that the flow is neutrally stable for all $\alpha$ when the slope of the base velocity profile is continuous across the interface. Since viscoelasticity does not change the base velocity profiles for the channel flow, the same neutral-stability curves exist for viscoelastic liquids as well. Although the flow of Newtonian liquids $\left(\Lambda_{1 j}=0\right)$ is unstable in the region of high $q_{2} / q_{1}$ and high $\alpha$, the flow of viscoelastic liquids $\left(\Lambda_{1 j} \neq 0\right)$ is stable in the same region. Although no flow of Newtonian liquids is stable for all $\alpha$ in the $\alpha-q_{2} / q_{1}$ plane, the flow of viscoelastic liquids is stable for all $\alpha$ at high flow-rate ratio $q_{2} / q_{1}$ in the same plane. This critical
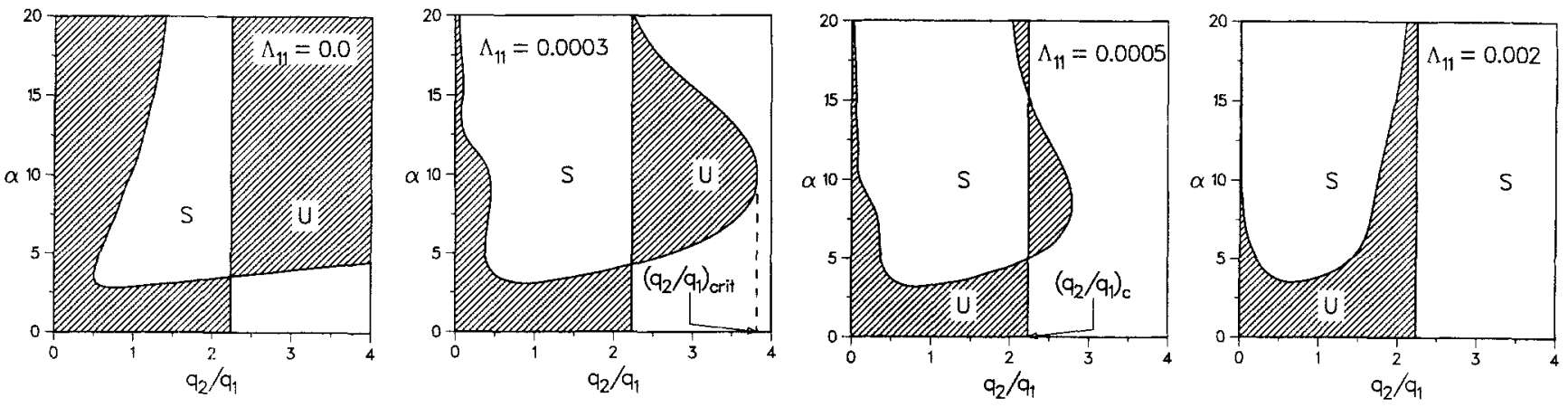

Figure 4. Neutral-stability curves for two-layer flow at various $\Lambda_{11}$.

Parameters: $R e_{1}=0.1 ; d_{j}=1 ; \kappa_{2}=5.0 ; \Lambda_{12} / \Lambda_{11}=0.1$

Hatched regions are unstable, unhatched regions are stable 

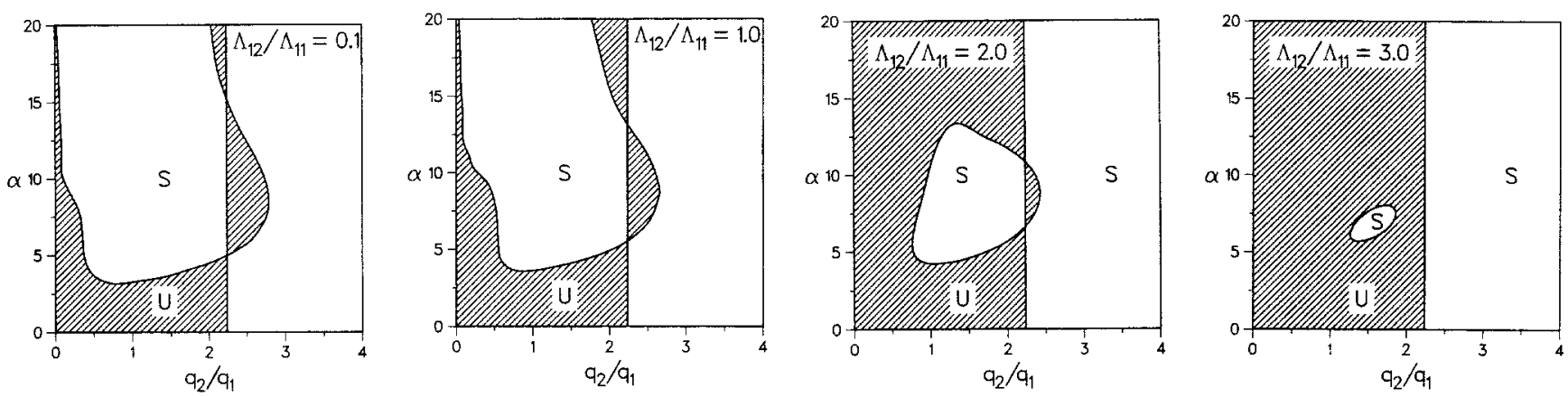

Figure 6. Neutral-stability curves for two-layer flow at various $\Lambda_{12} / \Lambda_{11}$.

Parameters: $R e_{1}=0.1 ; \kappa_{2}=5.0 ; \Lambda_{11}=0.0005$

Hatched regions are unstable, unhatched regions are stable

flow-rate ratio, denoted by $\left(q_{2} / q_{1}\right)_{\text {crii }}$, beyond which the flow is stable for all $\alpha$, reduces with increasing $\Lambda_{11}$. However, for $\Lambda_{11}>$ 0.001 , the stable region at high $q_{2} / q_{1}$ is unaltered. Therefore, $\left(q_{2} / q_{1}\right)_{c r i t}$ remains constant for $\Lambda_{1 j}>0.001$ and is equal to $\left(q_{2} / q_{1}\right)_{c}$. Thus, for the liquids of high elasticity, the critical flow-rate ratio can be estimated by the asymptotic analysis at $\alpha \rightarrow 0$. Since realistic disturbances incorporate a spectrum of $\alpha$, a stable operation of multilayer extrusion is possible only for $q_{2} / q_{1}>\left(q_{2} / q_{1}\right)_{\text {crit }}$. Thus, truly stable operating windows exist
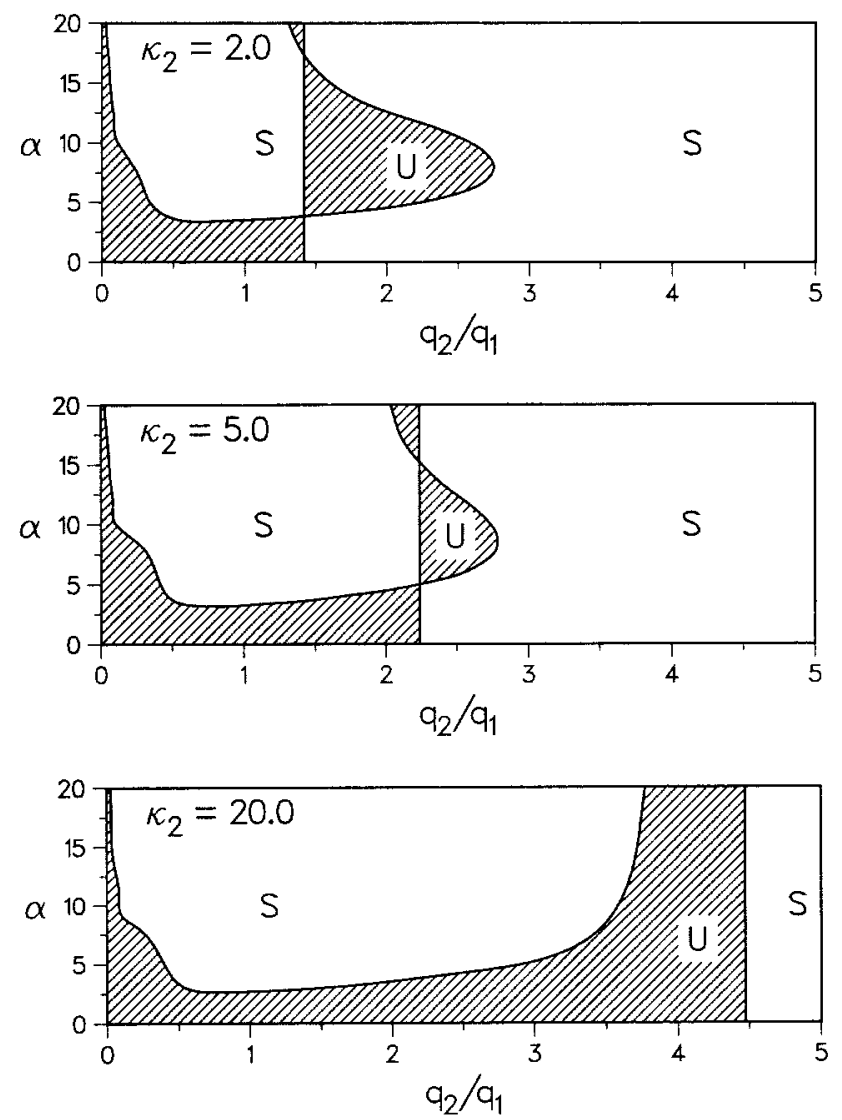

Figure 7. Neutral-stability curves for two-layer flow at various $\kappa_{2}$.

Parameters: $R e_{1}=0.1 ; d_{1}=1 ; \Lambda_{11}=0.0005 ; \Lambda_{12} \Lambda_{11}=0.1$ Hatched regions unstable, unhatched regions are stable for multilayer extrusion of viscoelastic liquids and not for that of Newtonian liquids. At sufficiently large $\alpha$ (or at sufficiently short wavelengths), the period of oscillation for the fluid particles following the path of flow with disturbance of wave number $\alpha$ is much smaller than the relaxation time of the fluid undergoing the disturbance. Therefore, the effect of the relaxation time dominates and returns the fluid to its original, undisturbed steady state. Since the flow is stable at low $\alpha$ and high $q_{2} / q_{1}$ as well, the combined effect is the existence of $\left(q_{2} / q_{1}\right)_{\text {crit }}$ beyond which the flow is stable at all $\alpha$ for any nonzero $\Lambda_{11}$. Similar effects take place in Figure 6 for increasing $\Lambda_{12} / \Lambda_{11}$.

The effect of $R e_{1}$ on the neutral-stability diagram of two-layer flow is shown in Figure 5 in the $\alpha-q_{2} / q_{1}$ plane. The parameters are $d_{j}=1, \kappa_{2}=5.0, \Lambda_{11}=0.002$, and $\Lambda_{12} / \Lambda_{11}=0.1$. As the Reynolds number increases, the unstable region at high $q_{2} / q_{1}$ expands. At low Reynolds number in Figure 5, $\left(q_{2} / q_{1}\right)_{\text {crit }}=$ $\left(q_{2} / q_{1}\right)_{c}$. However, with increasing Reynolds number, $\left(q_{2} / q_{1}\right)_{\text {crit }}$ increases substantially and is no longer equal to $\left(q_{2} / q_{1}\right)_{c}$. Since the Reynolds numbers affect the neutral-stability curves, the density ratios affect the neutral-stability curves as well, even in the absence of gravity. The interfacial instability is observed even at low speeds in industrial operations. Besides, the stable flow becomes unstable with increasing speed (Schrenk and

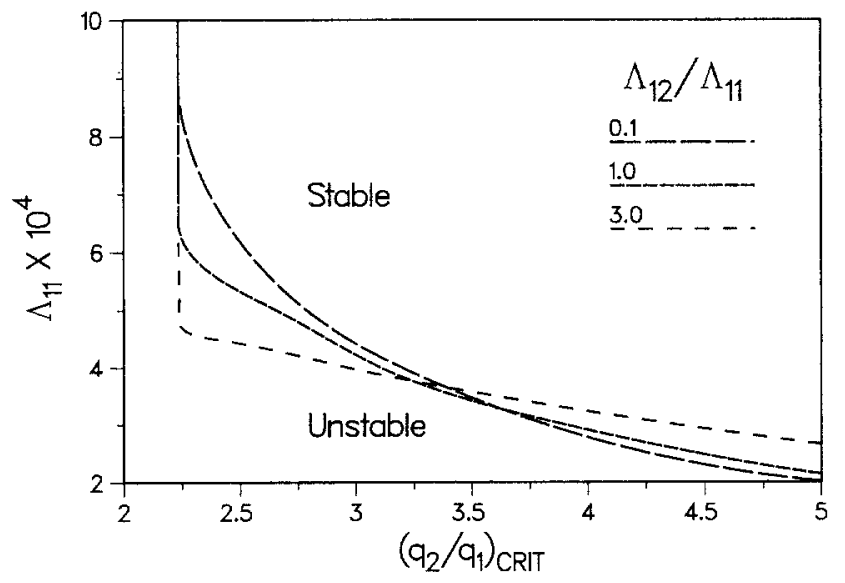

Figure 8. Operating diagrams of stable conditions at all $\alpha$ for two-layer flow for various $\Lambda_{12} / \Lambda_{11}$ in $\Lambda_{11}-$ $\left(q_{2} / q_{1}\right)_{c r i}$ plane.

Parameters: $\kappa_{2}=5.0 ; R e_{1}=0.1$ 


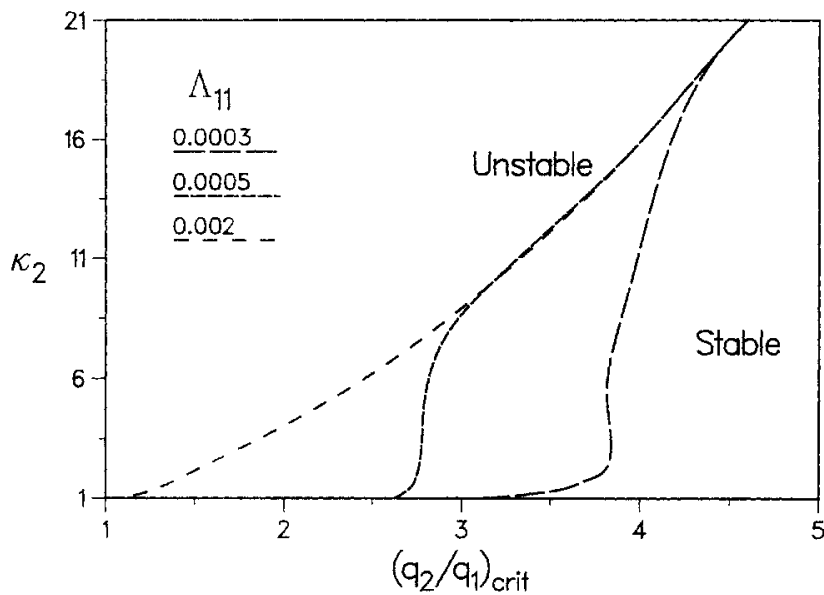

Figure 9. Operating diagrams of stable conditions at all $\alpha$ for two-layer flow for various $\Lambda_{11}$ in $\kappa_{2}-\left(q_{2} /\right.$ $\left.q_{1}\right)_{c r i t}$ plane.

Parameters: $\Lambda_{12} / \Lambda_{11}=0.1 ; d_{j}=1 ; R e_{1}=0.1$

Alfrey, 1978). Both of these observations agree with the results in Figure 5, which clearly shows that the instability may exist at low $\operatorname{Re}_{1}$, below $\left(q_{2} / q_{1}\right)_{\text {crit }}$, and that $\left(q_{2} / q_{1}\right)_{\text {crit }}$ increases with increasing $R e_{1}$. Note that the Reynolds number $\left(R e_{1}<20\right)$ does not influence the neutral-stability curves either of Newtonian liquids at any wavelengths (Yiantsios and Higgins, 1988) or those of viscoelastic liquids at $\alpha \rightarrow 0$. The influence of the Reynolds number on the neutral-stability curves of viscoelastic liquids can be attributed to the competing effects of viscoelastic and inertial forces, and increasing $\Lambda_{11}$ is similar to decreasing $R e_{1}$ in regard to the stability of two-layer flow. Since industrial extrusion processes of polymer melts are carried out at low speeds and since the $R e_{j}$ are defined in terms of the thickness $H$ of the die, the Reynolds number $R e_{1}$, chosen for all further calculations, equals 0.1 . The results do not change qualitatively for different values of Reynolds number.

The effect of the relaxation time of the second layer $\Lambda_{12}$ on the neutral-stability curves in the $\alpha-q_{2} / q_{1}$ plane is shown in Figure 6. The fixed parameters are $\Lambda_{11}=0.0005, R e_{1}=0.1, d_{j}=1$, and $\kappa_{2}=5.0$. Although at low $\Lambda_{12} / \Lambda_{11}$ the flow is stable in the region of high $\alpha$ and low $q_{2} / q_{1}$, this stable region shrinks with increasing $\Lambda_{12} / \Lambda_{11}$. At very high $\Lambda_{12} / \Lambda_{11}$ this stable region disappears. Therefore, the critical flow-rate ratio $\left(q_{2} / q_{1}\right)_{c r i t}$ reduces with increasing $\Lambda_{12} / \Lambda_{11}$, and for $\Lambda_{12} / \Lambda_{11}>2.2,\left(q_{2} /\right.$ $\left.q_{1}\right)_{\text {crit }}$ remains unaltered and corresponds to the asymptotic solution at $\alpha \rightarrow 0$. At very high $\Lambda_{12} / \Lambda_{11}$, there are only two regions in the $\alpha-q_{2} / q_{1}$ plane: the unstable region at low $q_{2} / q_{1}$ and the stable region at high $q_{2} / q_{1}$.

The effect of the viscosity of the second layer $\kappa_{2}$ is shown in Figure 7. The other parameters are $R e_{1}=0.1, d_{j}=1, \Lambda_{11}=$ 0.0005 , and $\Lambda_{12} / \Lambda_{11}=0.1$. Since $\left(q_{2} / q_{1}\right)_{c}=\sqrt{\kappa_{2}},\left(q_{2} / q_{1}\right)_{c}$ increases with increasing $\kappa_{2}$. With increasing $\kappa_{2},\left(q_{2} / q_{1}\right)_{\text {crit }}$ increases as well, and at high $\kappa_{2},\left(q_{2} / q_{1}\right)_{c r i t}=\left(q_{2} / q_{1}\right)_{c}$. This destabilizing effect of the viscosity stratification is similar to the one in the flow of Newtonian liquids (Yih, 1967; Yiantsios and Higgins, 1988; Anturkar et al,, 1990). Since by interchanging layers the same neutral stability curve is recovered, the flows with $\kappa_{2}<1.0$ are not investigated. Thus, industrial practice of matching viscosities to reduce instability is well justified by the analysis of two-layer flow.

All the above results can be presented concisely in the form of operating diagrams that identify the stable regions at all $\alpha$. One such operating diagram is shown in Figure 8 in the $\Lambda_{11}-$ $\left(q_{2} / q_{1}\right)_{\text {crit }}$ plane for various values of $\Lambda_{12} / \Lambda_{11}$. The liquid in the second layer is more viscous than the liquid in the first layer $\left(\kappa_{2}=5.0\right)$, the Reynolds number $R e_{1}$ is 0.1 , and the density ratio $d_{j}$ is 1 . The relaxation time $\Lambda_{11}$ stabilizes the flow for all $\Lambda_{12} / \Lambda_{11}$. All the curves at various $\Lambda_{12} / \Lambda_{11}$ merge into the curve of constant critical flow-rate ratio, which corresponds to the asymptotic solution at $\alpha \rightarrow 0$. The approach to the asymptotic solution is faster for higher $\Lambda_{12} / \Lambda_{11}$. The ratio $\Lambda_{12} / \Lambda_{11}$ stabilizes the flow for approximately $q_{2} / q_{1} \leq 3.4$ and destabilizes the flow for $q_{2} / q_{1}>3.4$. The stabilizing effect at low $q_{2} / q_{1}$ is more prominent than the destabilizing effect at high $q_{2} / q_{1}$. Thus, the effect may be stabilizing or destabilizing at low elasticities; at high elasticities, however, the stability is infiuenced only by the viscosity ratio, not by the elasticities. When the operating diagram is constructed in the $\kappa_{2}-\left(q_{2} / q_{1}\right)_{c r i t}$ plane for various values of $\Lambda_{11}$ and for $\Lambda_{12} / \Lambda_{11}=0.1$, Figure $9, \kappa_{2}$ destabilizes the flow and $\Lambda_{11}$ stabilizes the flow. All the curves merge into the curve of $\Lambda_{11}=0.002$ at high $\kappa_{2}$. For $\Lambda_{11}>0.002$, the curve remains unchanged as it corresponds to the asymptotic solution at $\alpha \rightarrow 0$.

The presence of an additional layer influences the stability diagram considerably. In Figure 10 the stability of three-layer flow rate is examined in the $\alpha-q_{2} / q_{1}$ plane for various values of the flow ratio $q_{3} / q_{1}$ and the relaxation time of the first layer $\Lambda_{11}$.
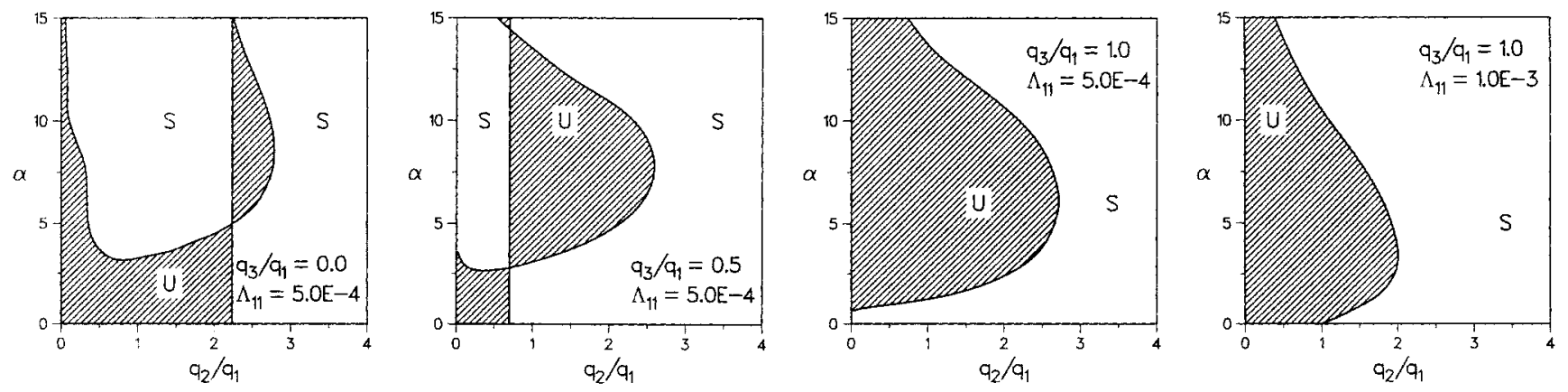

Figure 10. Neutral-stability curves for three-layer flow at various $q_{3} / q_{1}$.

Parameters: $\operatorname{Re} e_{1}=0.1 ; d_{j}=1 ; \kappa_{2}=5.0 ; \kappa_{3}=1.0 ; \Lambda_{12} / \Lambda_{11}=0.1 ; \Lambda_{13} / \Lambda_{11}=1.0$ Hatched regions are unstable, unhatched regions are stable 
The outer layers have the same material properties $\left(\kappa_{3}=\right.$ $\left.1.0, \Lambda_{13} / \Lambda_{11}=1.0\right)$ and the core layer is more viscous than the outer layer $\left(\kappa_{2}=5.0\right)$ with low relaxation time $\left(\Lambda_{12} / \Lambda_{11}=0.1\right)$. The relaxation time of the first layer is $\Lambda_{11}=0.0005$ in Figures $10 \mathrm{a}$ to $10 \mathrm{c}$. As $q_{3} / q_{1}$ increases, the neutral-stability curve of constant $q_{2} / q_{1}$ shifts to lower $q_{2} / q_{1}$. In other words, with increasing $q_{3} / q_{1},\left(q_{2} / q_{1}\right)_{c}$ decreases, and at $q_{3} / q_{1}=1.0$, $\left(q_{2} / q_{1}\right)_{c}=0.0$. The critical flow-rate ratio $\left(q_{2} / q_{1}\right)_{c r i t}$ decreases with increasing $q_{3} / q_{1}$ from 0.05 to 0.5 , and then increases again as $q_{3} / q_{1}$ increases from 0.5 to 1.0 . In Figure $10 \mathrm{~d}$, the neutralstability curve is plotted for $q_{3} / q_{1}=1.0$ and $\Lambda_{11}=0.001$. The flow-rate ratio $q_{2} / q_{1}$ at small $\alpha$ increases with increasing $\Lambda_{11}$, Figure 2. However, $\left(q_{2} / q_{1}\right)_{\text {crit }}$ decreases with increasing $\Lambda_{11}$. The calculations at higher $\Lambda_{11}$ show that at certain $\Lambda_{11}>0.001$, $\left(q_{2} / q_{1}\right)_{\text {crit }}$ corresponds to the asymptotic solution and then with increasing $\Lambda_{11},\left(q_{2} / q_{1}\right)_{c r i t}$ increases and reaches $\left(q_{2} / q_{1}\right)_{c}$.

Similar effects on the neutral-stability diagram were also observed by varying the relaxation time of the liquid in the third layer, Figure 11. The parameters are $\Lambda_{11}=0.0005, \Lambda_{12} / \Lambda_{11}=$ $0.1, \kappa_{2}=5.0, \kappa_{3}=1.0, R e_{1}=0.1, d_{j}=1$, and $q_{3} / q_{1}=1.0$. With increasing $\Lambda_{13} / \Lambda_{11}$ from 0.1 to $3.0,\left(q_{2} / q_{1}\right)_{\text {crit }}$ decreases sharply. However, by increasing $\Lambda_{13} / \Lambda_{11}$ further, the critical flow-rate ratio $\left(q_{2} / q_{1}\right)_{\text {crit }}$ corresponds to the asymptotic solution and increases with increasing $\Lambda_{13} / \Lambda_{11}$.

The viscosity ratio of the third layer to the first layer $\kappa_{3}$ also affects the critical flow-rate ratio significantly, as shown in Figure 12. The fixed parameters are $\kappa_{2}=5.0, q_{3} / q_{1}=1.0$, $R e_{1}=0.1, d_{j}=1, \Lambda_{11}=0.0005, \Lambda_{12} / \Lambda_{11}=0.1$, and $\Lambda_{13} / \Lambda_{11}=$ 1.0. At low $\kappa_{3},\left(q_{2} / q_{1}\right)_{\text {crit }}$ is very high. However, as $\kappa_{3}$ increases, $\left(q_{2} / q_{1}\right)_{c r i t}$ decreases. At high $\kappa_{3},\left(q_{2} / q_{1}\right)_{c r i t}$ corresponds to the asymptotic solution. As $\kappa_{3}$ increases from 0.3 to $1.0,\left(q_{2} / q_{1}\right)_{c}$ decreases and at $\kappa_{3}=1.0,\left(q_{2} / q_{1}\right)_{c}=0.0$. However, as $\kappa_{3}$ increases further, $\left(q_{2} / q_{1}\right)_{c}$ increases. Thus, in three-layer flow, optimum values of $\Lambda_{11}, \Lambda_{13}$, and $\kappa_{3}$ exist for which $\left(q_{2} / q_{1}\right)_{\text {crit }}$ is a minimum.

To emphasize the destabilization of the symmetric flow configuration of three-layer flow, the operating diagram is constructed in the $\kappa_{3}-\left(q_{2} / q_{1}\right)_{\text {crit }}$ plane for various values of $\Lambda_{11}$ in Figure 13 that defines the stable regions at all $\alpha$. At very low $\Lambda_{11}$, the stability improves with increasing $\kappa_{3}$. As $\Lambda_{11}$ increases, the stability improves at low $\kappa_{3}$ except near $\kappa_{3}=1.0$. A sharp expansion in the unstable region near $\kappa_{3}=1.0$ resembles that in the asymptotic analysis and indicates that the symmetric flow configuration in the three-layer flow is unstable when the
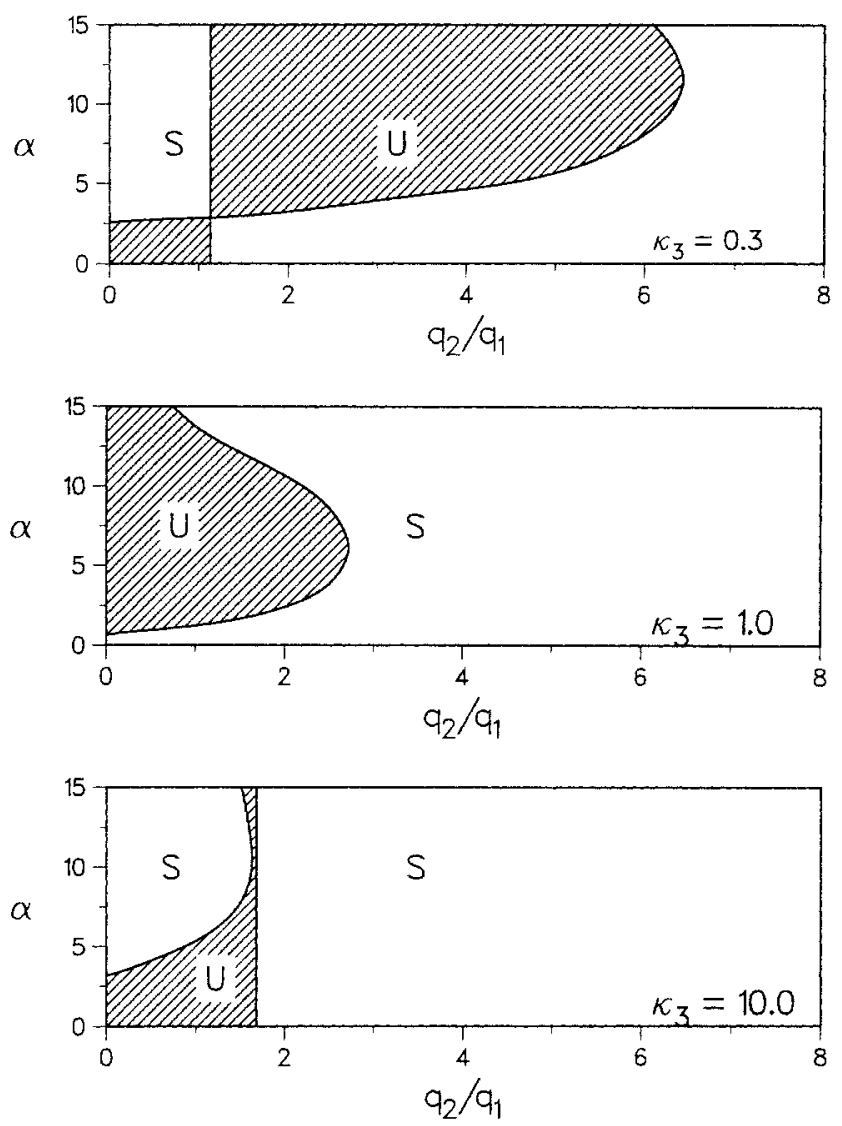

Figure 12. Neutral-stability curves for three-layer flow at various $\kappa_{\mathbf{3}}$.

$R e_{1}=0.1 ; d_{j}=1 ; \kappa_{2}=5.0 ; \Lambda_{11}=0.0005 ; \Lambda_{12} / \Lambda_{11}=0.1 ;$ $\Lambda_{33} / \Lambda_{11}=1.0 ; q_{3} / q_{1}=1.0$

Hatched regions are unstable, unhatched regions are stable

core layer is more viscous. At very high $\kappa_{3}$, all curves merge as $\left(q_{2} / q_{1}\right)_{\text {crit }}$ corresponds to the asymptotic solution, which is independent of $\Lambda_{11}$. There are two modes associated with two interfaces that can become unstable in three-layer flow. A sharp expansion of the unstable region about the symmetric configuration may be attributed to the resonance effect between the two free waves at the two interfaces. Similar resonance effects were observed by $\mathrm{Li}$ (1969a) in symmetric three-layer Couette
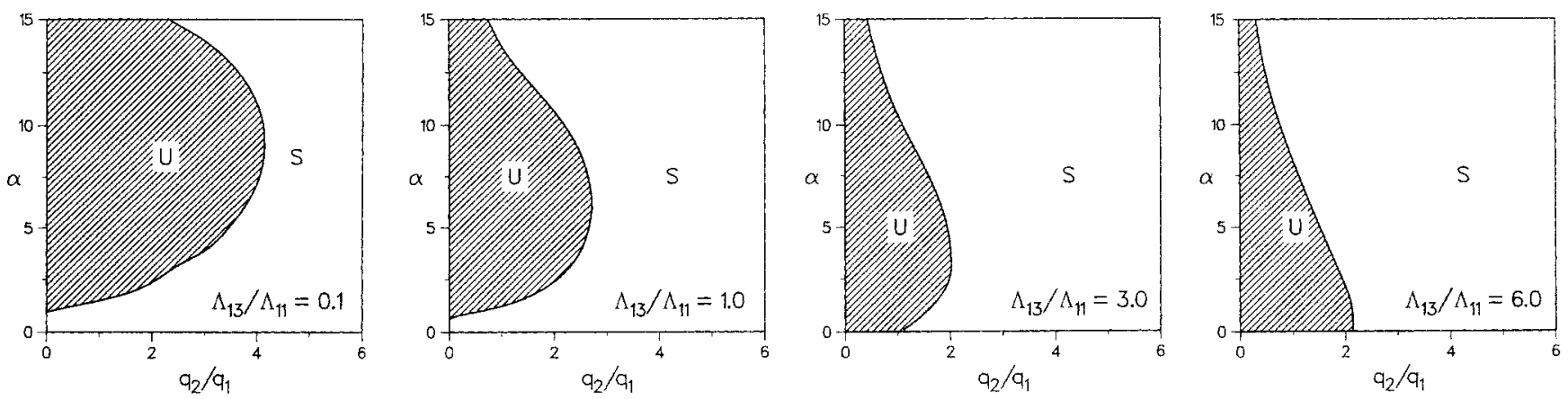

Figure 11. Neutral-stability curves for three-layer flow at various $\Lambda_{13} / \Lambda_{11}$.

Parameters: $R e_{1}=0.1, d_{j}=1 ; \kappa_{2}=5.0 ; \kappa_{3}=1.0 ; \Lambda_{11}=0.0005 ; \Lambda_{12} / \Lambda_{11}=0.1 ; q_{3} / q_{1}=1.0$.

Hatched regions are unstable, unhatched regions are stable 


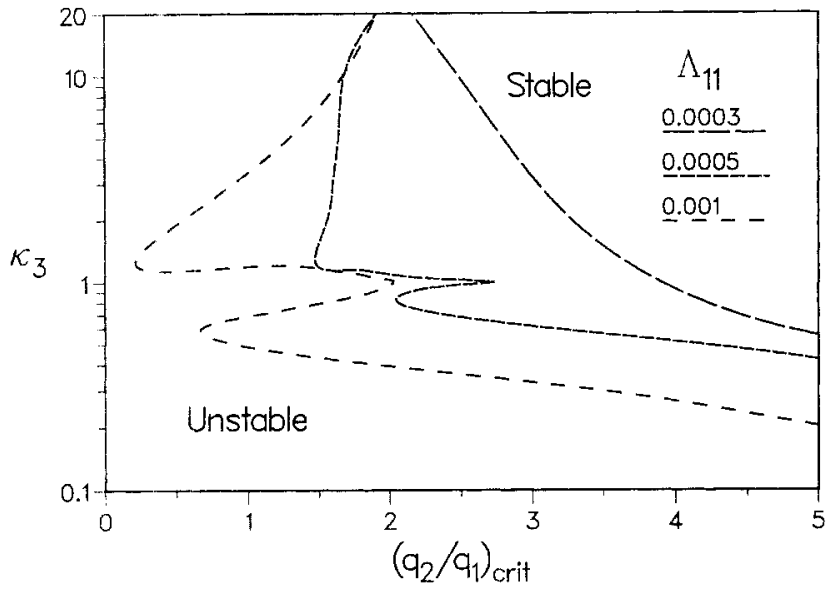

Figure 13. Operating diagrams of stable conditions at all $\alpha$ for three-layer flow for various $\Lambda_{11}$ in $\kappa_{3}-$ $\left(q_{2} / q_{1}\right)_{\text {crit }}$ plane.

Parameters: $R e_{1}=0.1 ; d_{j}=1 ; \kappa_{2}=5.0: \Lambda_{12} / \Lambda_{11}=0.1 ;$ $\Lambda_{13} / \Lambda_{13}=1.0$;

flow of Newtonian liquids. In fact, at $\kappa_{3}=1.0$, the configuration is symmetric and both the modes associated with the two interfaces are identical. When $\kappa_{3}$ departs from 1 , one mode becomes more unstable than the other and the switching of the dominant mode occurs at the symmetric configuration. When recycled plastic scrap is introduced in only one of the outer layers, the layers are no more symmetric, and in those cases, the stability of the process is observed to be sensitive to this geometrical symmetry (private communication, W. J. Schrenk, Dow Chemical Co., 1988).

\section{Liquids with shear rate dependent viscosity}

From earlier discussion of the constitutive equation, there are two parameters in the shear rate dependent Carreau viscosity function that characterize the shear-thinning behavior of viscosity. They are the time constant $\Lambda_{v j}$, which signifies the onset of shear thinning, and the power-law exponent $n_{j}$, which determines the degree of shear thinning. As $\Lambda_{v j}$ increases, the minimum shear rate at which the power-law behavior is exhibited by the viscosity function decreases. As the power-law exponent $n_{j}$ decreases, shear thinning increases.

In Figure 14, the neutral-stability curves of two-layer flow are plotted in the $\alpha-q_{2} / q_{1}$ plane for various values of $\Lambda_{v j}$ at $n_{1}=$ $n_{2}=0.7$. The fixed parameters are $R e_{1}=0.1, d_{j}=1, \kappa_{2}=5.0$, $\Lambda_{11}=0.001, \Lambda_{12} / \Lambda_{11}=0.1$, and $\Lambda_{v 2}=\Lambda_{v 1}$. At $\Lambda_{v j}=0.0$, the liquids have constant viscosity and as $\Lambda_{v j}$ increases, the liquids near the walls start exhibiting shear-thinning behavior. The flow domain in which shear-thinning behavior is exhibited by the liquids expands with increasing $\Lambda_{v j}$. From Figure 14, $\left(q_{2} / q_{1}\right)_{c}$ increases gradually with increasing $\Lambda_{t j}$. The calculations also indicate that the location of the steady-state interface is identical to the zero shear rate location at $\left(q_{2} / q_{1}\right)_{c}$. When the location of the interface is different from the zero shear rate location, the actual viscosity ratio at the interface is always higher than the zero shear rate viscosity ratio. Since the flow-rate ratio $q_{2} / q_{1}$, at which the flow is neutrally stable at $\alpha \rightarrow 0$, is minimum at the zero shear viscosity ratio, the location of the interface matches with the location of the zero shear rate at $\left(q_{2} / q_{1}\right)_{c}$. The critical flow-rate ratio $\left(q_{2} / q_{1}\right)_{\text {crii }}$, however, increases significantly with increasing $\Lambda_{v j}$. At very high values of $\Lambda_{v j}$ the viscosity function exhibits power-law behavior over almost the complete domain of flow, and further increase in $\Lambda_{v j}$ does not change the behavior of the viscosity function significantly. Therefore, at very high $\Lambda_{v j}$ the increase in $\left(q_{2} / q_{1}\right)_{\text {crit }}$ is insignificant.

The effect of the power-law exponent is also studied in Figure 15. The neutral-stability curves of two-layer flow are plotted in the $\alpha-q_{2} / q_{1}$ plane for various values of $n_{j}$. The fixed parameters are $R e_{1}=0.1, d_{j}=1, \kappa_{2}=5.0, \Lambda_{11}=0.001$, $\Lambda_{12} / \Lambda_{11}=0.1, \Lambda_{v j}=1.0$, and $n_{1}=n_{2}$. For a given $\Lambda_{v j}$, there are three factors that contribute to the sharp destabilizing effect of decreasing $n_{j}$, as observed in Figure 15:

1. Shear-thinning behavior exists at lower shear rate for lower $n_{j}$

2. The slope of the viscosity vs. shear rate on a log-log scale increases for decreasing $n_{j}$

3. The viscosity ratio at the interface increases with decreasing $n_{j}$ as it departs from the zero shear rate location

The asymptotic solution $\left(q_{2} / q_{1}\right)_{c}$, however, does not change as sharply as $\left(q_{2} / q_{1}\right)_{\text {crit }}$.

The destabilizing effect of the shear-thinning degree increases with the zero shear rate viscosity ratio, as shown in Figure 16a. In this figure, the stability results are plotted in the form of an operating diagram in the $\kappa_{2}-\left(q_{2} / q_{1}\right)_{c r i t}$ plane, which defines the stable regions at all $\alpha$. The other parameters are $R e_{1}=0.1$, $d_{j}=1, \kappa_{2}=5.0, \Lambda_{11}=0.001, \Lambda_{12} / \Lambda_{11}=0.1$, and $\Lambda_{v j}=1.0$. The destabilizing effect of increasing $\kappa_{2}$ is more pronounced at lower
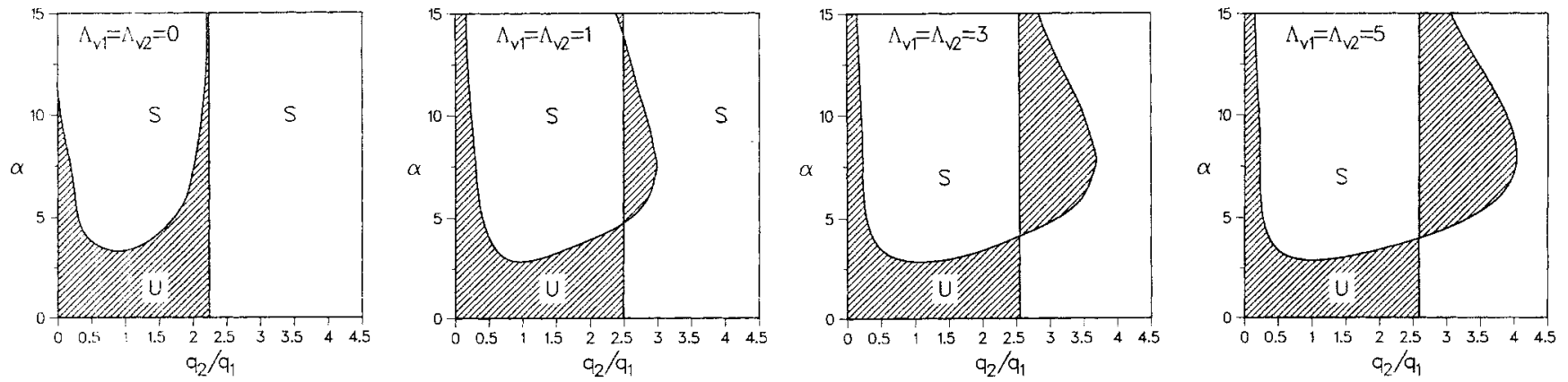

Figure 14. Neutral-stability curves for two-layer flow at various $\Lambda_{n}=\Lambda_{n^{2}}$.

Parameters: $\operatorname{Re}_{1}=0.1 ; d_{j}=1 ; \kappa_{2}=5.0 ; \Lambda_{11}=0.001 ; \Lambda_{12} / \Lambda_{11}=0.1 ; n_{1}=n_{2}=0.7$

Hatched regions are unstable, unhatched regions are stable 

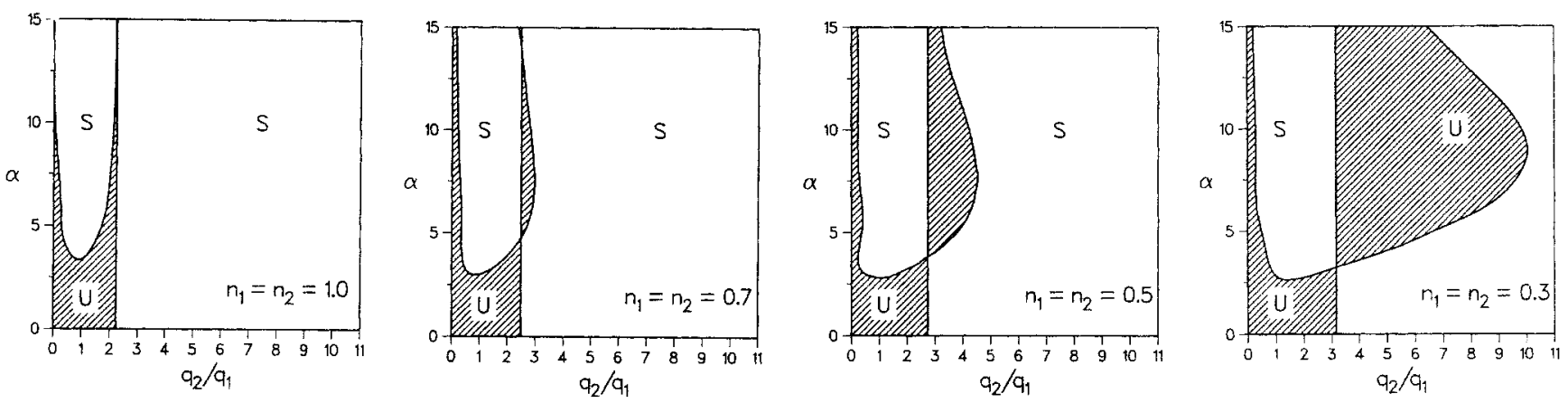

Figure 15. Neutral-stability curves for two-layer flow at various $n_{1}=\boldsymbol{n}_{2}$.

Parameters: $R e_{1}=0.1 ; d_{i}=1 ; \kappa_{2}=5.0 ; \Lambda_{11}=0.001 ; \Lambda_{12} / \Lambda_{11}=0.1 ; \Lambda_{i 1}=\Lambda_{r 2}=1.0$

Hatched regions are unstable, unhatched regions are stable

$n_{j}$. The viscosity ratio at the interface is higher than the zero shear rate viscosity ratio except when the interface is located at the zero shear rate location. Therefore, to separate the effects due to shear-thinning behavior and due to the viscosity ratio at

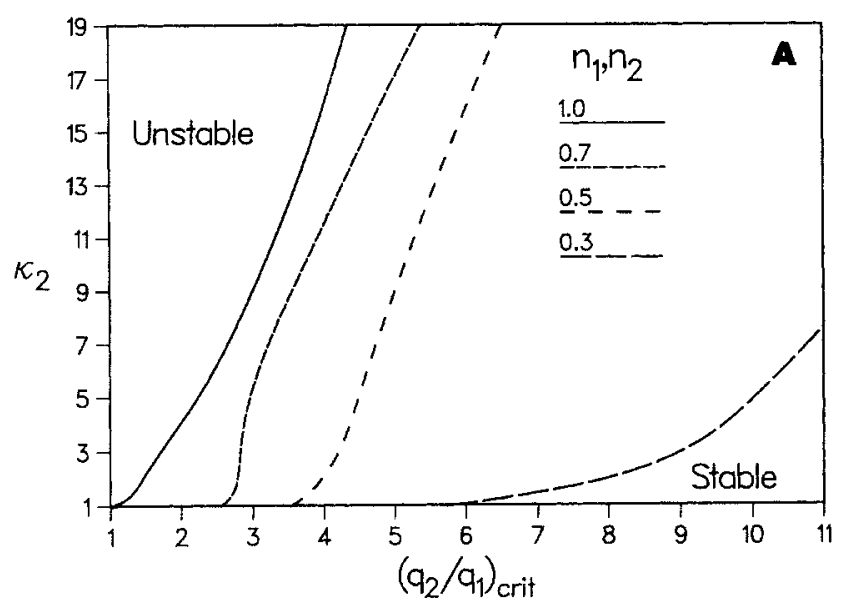

a. In plane of zero shear rate viscosity ratio $\kappa_{2}$ and flow rate ratio $\left(q_{2} / q_{1}\right)_{\text {crir }}$

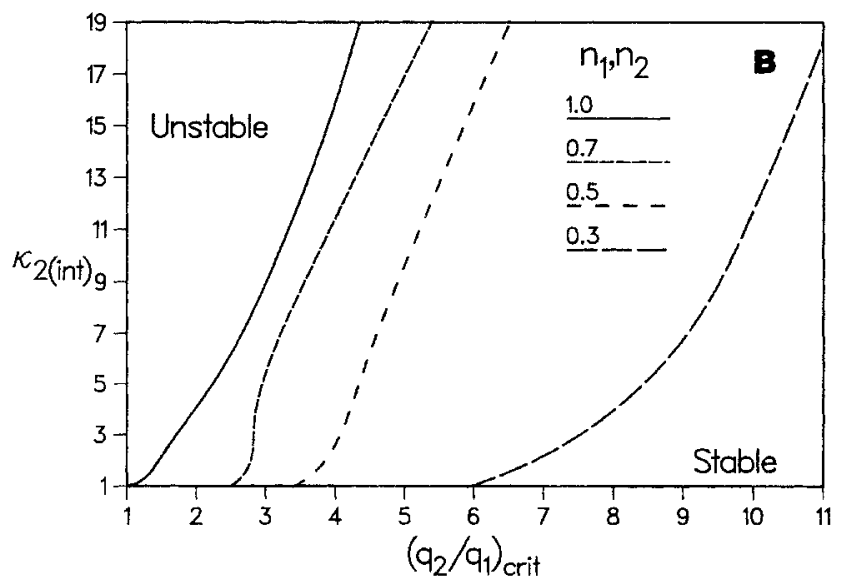

b. In plane of viscosity ratio at interface $\kappa_{2(i n)\}}$ and fow rate ratio $\left(q_{2} / q_{1}\right)_{c r i}$

Figure 16. Operating diagrams of stable conditions at all $\alpha$ for two-layer flow for various $n_{1}=n_{2}$. Parameters: $\Lambda_{11}=0.001 ; \Lambda_{12} / \Lambda_{11}=0.1 ; \Lambda_{t 1}=\Lambda_{v 2}=1.0$; $\operatorname{Re}_{1}=0.1 ; d_{j}=1$ the interface, the operating diagram is constructed in Figure $16 \mathrm{~b}$ in the plane of the viscosity ratio at the interface $\kappa_{2(i \mathrm{in})}$ and the critical flow rate ratio $\left(q_{2} / q_{1}\right)_{\text {crit }}$. The parameters are the same as those in Figure 16a. Although the destabilizing effect is not as pronounced as in Figure 16a, the unstable region still expands significantly with decreasing $n_{j}$. Thus, the shearthinning behavior itself does contribute in destablizing the flow.

Similar destabilizing effects of the shear-thinning behavior are observed for three-layer flow. Therefore, the neutralstability diagrams of three-layer flow are not presented here.

\section{Conclusions}

A two-dimensional linear stability analysis of multilayer plane Poiseuille flow of viscoelastic liquids has been conducted. As an inherent part of the study, a steady-state analysis was also implemented. The constitutive equation used is the modified Oldroyd-B model with shear rate dependent viscosity, represented by the Carreau viscosity function. The asymptotic analysis at long-wavelength disturbances and the numerical analysis at wavelengths of $O(1)$ were carried out. Results were presented for two- and three-layer flows under negligible gravity and interfacial tensions.

The relaxation and retardation times do not play any role in determining the neutral-stability curves at long-wavelength disturbances in two-layer flow, whereas additional neutralstability curves at high elasticity are observed near symmetric flow configurations, apart from the neutral-stability curves that are identical to those of Newtonian liquids. The relaxation and retardation times alter the neutral-stability diagrams considerably at wavelengths of $O(1)$. Viscosity stratification and inertial effects are primarily responsible for the onset of instability. Unlike in flows of Newtonian liquids, where the Reynolds number does not influence the neutral-stability curves at low Reynolds number, in flows of viscoelastic liquids increasing Reynolds number destabilizes the flow, even at low Reynolds number. Although the neutral-stability diagrams of Newtonian liquids do not have stable regions that persist at all wavelengths, the neutral-stability diagrams of viscoelastic liquids do have regions that are stable at all wavelengths. Therefore, it is possible to construct diagrams in which stable operating conditions at all wavelengths can be identified for viscoelastic liquids.

The instability is enhanced by increasing the zero shear rate viscosity ratio in two-layer flow. However, in three-layer flow, the zero shear rate viscosity ratio of the third layer to the first layer $\left(\kappa_{3}\right)$ stabilizes the flow at lower values of $\kappa_{3}$ and destabilizes 
the flow at higher values of $\kappa_{3}$. In three-layer flow, when the core layer is more viscous than the outer layers, increasing flow rate ratio of the third layer to the first layer stabilizes the flow except for symmetric and nearly symmetric configurations. Increasing relaxation time enhances stability up to a certain critical limit. Beyond this limiting value of relaxation time, however, elasticity does not influence the stability and the critical flow-rate ratio is determined by the asymptotic analysis at long wavelengths. Shear thinning always destabilizes the flow. The effect is more dramatic with the variation in the power-law exponent, rather than with the variation in the onset of shear thinning.

The importance of the asymptotic analysis at long wavelengths is highlighted by the above results. The asymptotic analysis can be performed quickly on small computers, and does provide the critical conditions for the stability of multilayer flow of liquids with high elasticity and with high viscosity ratios. The neutral-stability curves obtained by the asymptotic analysis are independent of the relaxation times in two-layer flow. However, the limits beyond which the asymptotic analysis is sufficient to obtain critical conditions for the stability, still need to be predicted by the analysis of wavelengths of $O(1)$.

Although the linear stability analysis can predict the onset of instability for infinitesimal disturbances, the stability to the finite disturbances cannot be studied. The study of finite disturbances is particularly important for industrial multilayer extrusion operations to determine whether the subcritical instability exists or not. Currently, we are conducting work to investigate the transient response to finite disturbances.

\section{Acknowledgment}

This research is being funded by a grant from the Dow Chemical Company, Midland, Michigan, whose support is gratefully acknowledged. We also wish to acknowledge valuable suggestions offered by $A$. P. Hooper

\section{Notation}

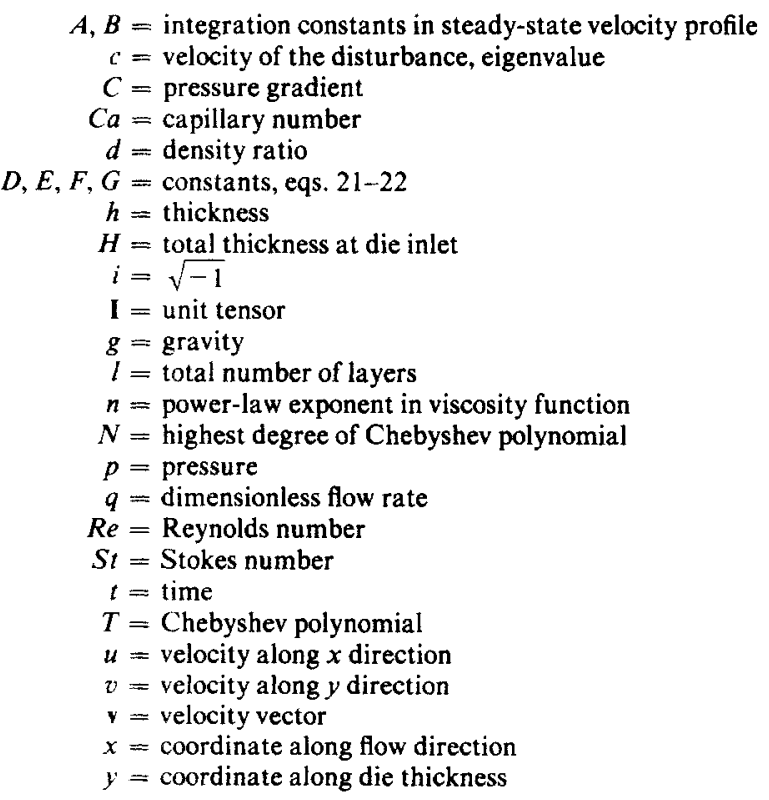

\section{Greek letters}

$$
\begin{aligned}
& \alpha=\text { wave number of the disturbance } \\
& \dot{\gamma}=\text { magnitude of rate-of-strain tensor } \\
& \dot{\gamma}=\text { rate-of-strain tensor }
\end{aligned}
$$

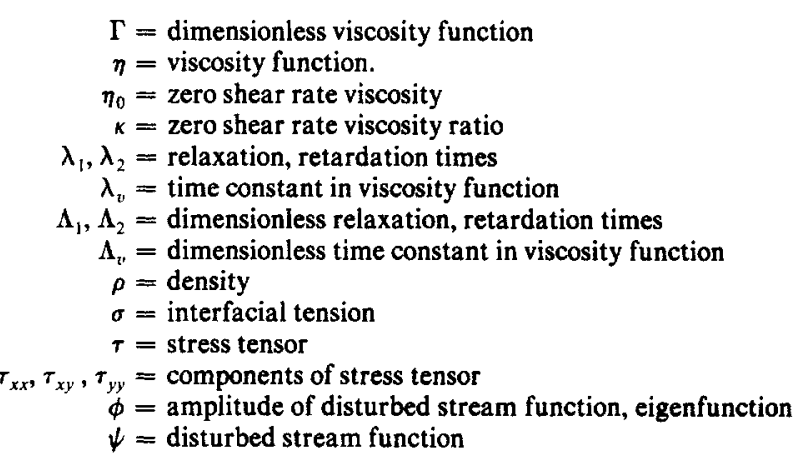

Superscripts

$\begin{aligned} \hat{-} & =\text { disturbance } \\ & =\text { amplitudes of the disturbance }\end{aligned}$

\section{Subscripts}

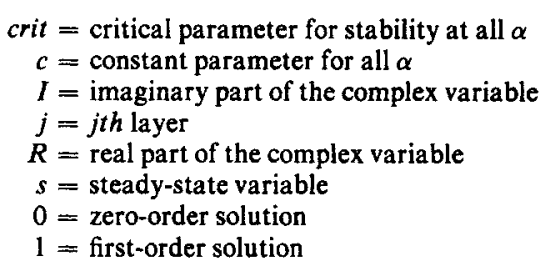

\section{Mathematical operations}

$\tau_{(1)}=$ convected derivative of tensor $\tau$, Eq. 2

$\dot{\boldsymbol{\gamma}}_{(2)}=$ convected derivative of tensor $\dot{\boldsymbol{\gamma}}, \mathrm{Eq} .3$

\section{Literature Cited}

Anturkar, N. R., T. C. Papanastasiou, and J. O. Wilkes, "Linear Stability Analysis of Multilayer Plane Poiseuille Flow," Phys. Fluids A, 2, 503 (1990).

Bird, R. B., R. C. Armstrong, and O. Hassager, Dynamics of Polymeric Liquids, 1: Fluid Mechanics, 2nd ed., Wiley, New York (1987).

Drazin, P. G., and W. H. Reid, Hydrodynamic Stability, Cambridge Univ. Press (1981).

Gottlieb, D., and S. A. Orszag, Numerical Analysis of Spectral Methods: Theory and Applications, CBMS-NSF Regional Conf. Ser. App. Math., 26, SIAM, Philadelphia (1977).

Han, C. D., Multiphase Flows in Polymer Processing, Academic Press, New York (1981).

Han, C. D., and R. Shetty, "Studies of Multilayer Film Coextrusion. II: Interfacial Instability in Flat Film Coextrusion," Polym. Eng. Sci., 18, 180 (1978).

Hooper, A. P., "The Stability of Two Superposed Fluids in a Channel," Phys. Fluids A, 1, 1133 (1989).

"Long-wave Instability at the Interface between Two Viscous Fluids: Thin Layer Effect," Phys. Fluids, 28, 1613 (1985).

Hooper, A. P., and W. G. C. Boyd, "Shear Flow Instability at the Interface between Two Viscous Fluids," J. Fluid Mech., 128, 507 (1983).

Kao, T. W., and C. Park, "Experimental Investigations of the Stability of Channel Flows. 2: Two-layered Cocurrent Flow in a Rectangular Channel," J. Fluid Mech., 52, 401 (1972).

Khan, A. A., and C. D. Han, "A Study on the Interfacial Instability in the Stratified Flow of Two Viscoelastic Fluids Through a Rectangular Duct," Trans. Soc. Rheol., 21(1), 101(1977).

Li, C. H., "Instability of Three-Layer Viscous Stratified Flow," Phys. Fluids, 12, 2473 (1969a).

_- "Stability of Two Superposed Elastoviscous Liquids in Plane Couette Flow," Phys, Fluids, 12, 531 (1969b).

Orszag, S. A., "Accurate Solution of the Orr-Sommerfeld Stability Equation," J. Fluid Mech., 50, 689 (1971).

Renardy, Y. "The Thin-layer Effect and Interfacial Stability in a Two-layer Couette Flow with Similar Liquids," Phys. Fluids, 30, 1627 (1987a). 
"Viscosity and Density Stratification in Vertical Poiseuille Flow," Phys. Fluids, 30, 1638 (1987b).

"Stability of the Interface in Two Layer Couette Flow of Upper Convected Maxwell Liquids," J. Non-Newt. Fluid Mech., 28, 180 (1988).

Schrenk, W. J., and T. Alfrey, "Coextruded Multilayer Polymer Films and Sheets," Polymer Blends, vol. 2, D. R. Paul and N. Seymour, eds., Academic Press, New York, 129 (1978).

Smith, M. K., and S. H. Davis, "The Instability of Sheared Liquid Layers," J. Fluid Mech., 12, 187 (1982).

Taylor, G. I., "Effect of Variation in Density on the Stability of Superposed Streams of Fluids," Proc. Roy. Soc., A132, 499 (1931).
Waters, N. D., "The Stability of Two Stratified 'Power-Law' Liquids in Couette Flow," J. Non-Newt. Fluid Mech., 12, 85 (1983).

Waters, N. D., and A. M. Keeley, "The Stability of Two Stratified Non-Newtonian Liquids in Couette Flow," J. Non-Newt. Fluid Mech., 24, 161 (1987)

Yiantsios, S. G., and B. G. Higgins, "Linear Stability of Plane Poiseuille Flow of Two Superposed Fluids: Shear and Interfacial Modes," Phys. Fluids, 31, 3225 (1988).

Yih, C. S., "Instability Due to Viscosity Stratification," J. Fluid Mech., 27, 337 (1967)

Manuscript received Nov. 8, 1989, and revision received Feb. 28, 1990. 